NASA Technical Memorandum 160741

\title{
The Sensitivity of Synthetic Aperture Radiometers for Remote Sensing Applications From Space
}

D.M. Le Vine

December 1989

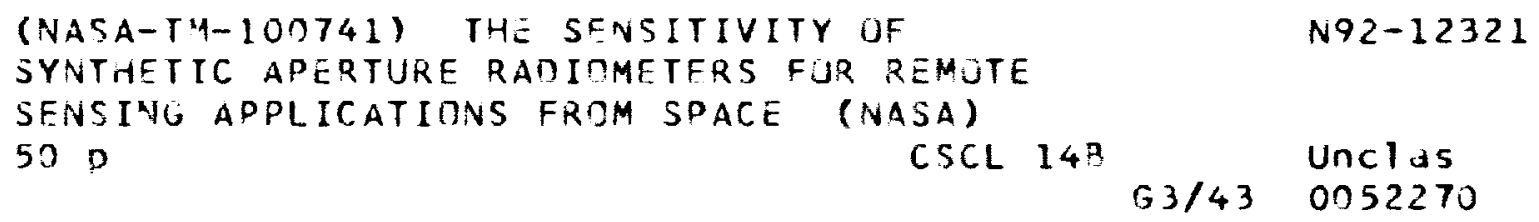

N92-12321 
t.

r

$-$

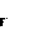

. 
NASA Technical Memorandum 100741

\section{The Sensitivity of Synthetic Aperture Radiometers for Remote Sensing Applications From Space}

D. M. Le Vine

Goddard Space Flight Center

Greenbelt, Maryland 


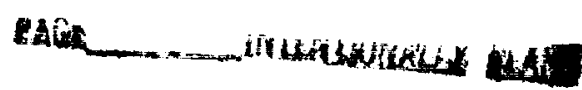




\section{CONTENTS}

Page

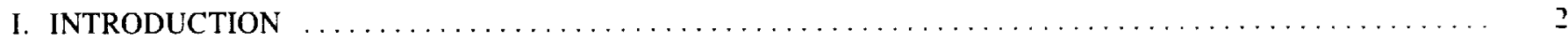

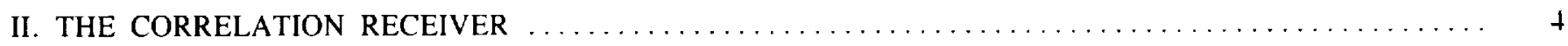

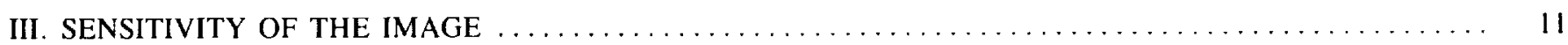

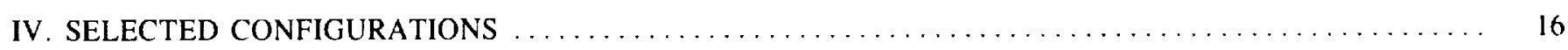

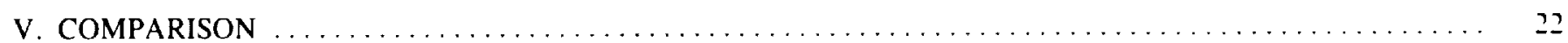

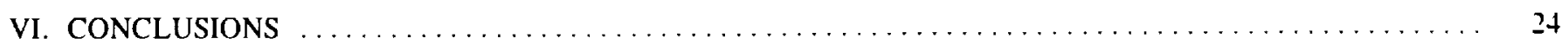

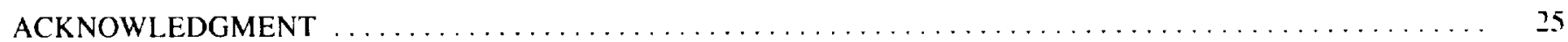

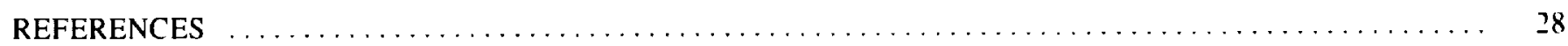

APPENDIX A. THE MEAN AND VARIANCE OF THE OUTPUT OF A CORRELATION RECEIVER $\ldots \ldots$ A-I

APPENDIX B. POWER SPECTRUM OF THE ANTENNA OUTPUT VOLTAGES $\ldots \ldots \ldots \ldots \ldots \ldots \ldots \ldots$ B.I

APPENDIX C. THE SAGITTAL APPROXIMATION $\ldots \ldots \ldots \ldots \ldots \ldots \ldots \ldots \ldots \ldots \ldots \ldots \ldots \ldots \ldots \ldots \ldots \ldots$

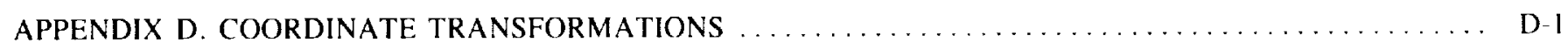

APPENDIX E. RELATIONSHIP BETWEEN BRIGHTNESS AND EI.ECTRIC FIEL.D INTENSITY $\ldots \ldots \ldots \ldots$ E-I

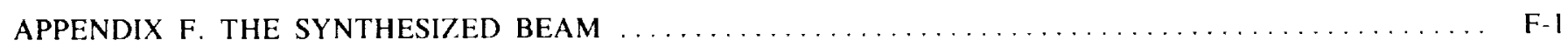




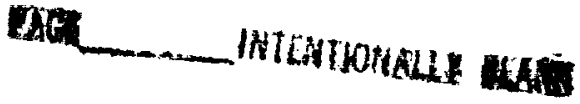




\section{THE SENSITIVITY OF SYNTHETIC APERTURE RADIOMETERS FOR REMOTE SENSING APPLICATION FROM SPACE}

by

David M. Le Vine

\section{PREFACE}

Aperture synthesis offers a means of realizing the full potential of microwave remote sensing from space by helping to overcome the limitations set by antenna size. The result is a potentially lighter, more adaptable structure for applications in space. However, because the physical collecting area is reduced, the signal-to-noise ratio is reduced and may adversely affect the radiometric sensitivity.

Sensitivity is an especially critical issue for measurements to be made from low earth orbit because the motion of the platform (about $7 \mathrm{~km} / \mathrm{s}$ ) limits the integration time available for forming an image.

The purpose of this paper is to develop expressions for the sensitivity of remote sensing systems which use aperture synthesis. The objective is to develop basic equations general enough to be used to obtain the sensitivity of the several variations of aperture synthesis which have been proposed for sensors in space. The conventional microwave imager (a scanning total power radiometer) is treated as a special case and the paper concludes with a comparison of three synthetic aperture configurations with the conventional imager. 


\section{INTRODUCTION}

Microwave remote sensing from space offers the potential for measuring many of the parameters important for understanding the environment of the earth on a global scale (Butler et al., 1988; Murphy et al., 1987), Among the parameters which can be measured are sea surface temperature (Wilheit and Chang, 1980), ocean salinity (Thomann, 1976), soil moisture (Wang et al., 1983), and sea ice concentration (Swift et al., 1985). Microwave remote sensing has the advantage that it can be done in the presence of cloud cover, permitting measurement of these parameters in regions inaccessible to visible and infrared sensors. Furthermore, because of the strong sensitivity of microwave radiation to the presence of water and the ability of microwave radiation to penetrate beneath the surface, unique information is available at microwave frequencies of compliment remote sensing at visible and infrared frequencies.

However, realizing the full potential of passive microwave remote sensing from space requires putting relatively large antennas in space. The apertures required for microwave sensors are large compared to those required of visible and infrared sensors because of the much longer wavelength in the microwave portion of the spectrum. For example, antenna size is the factor limiting the implementation of an L-band radiometer in space to measure soil moisture, and antenna size is an important factor limiting development of a microwave sensor to fill the gaps created by clouds in present-day visible and infrared soundings of the atmosphere.

A possible means of overcoming this size limitation is to use aperture synthesis (e.g., Le Vine and Good, 1983; Thompson, Moran and Swenson, 1986). This is a technique in which correlation receivers are used to coherently measure the product of the signal from pairs of antennas with many different antenna spacings. For distant sources, it can be shown that this correlation function is proportional to the Fourier transform of the intensity of the source at a frequency which depends on the spacing between the antennas. By making measurements at many different spacings one determines the Fourier spectrum of the source; then a map of the source can be obtained after all measurements are complete by inverting the transform. The resolution obtained in this manner is determined by how well the correlation function has been measured, not by the size of the antennas used. In principle, one can obtain very high-resolution maps of the source by measuring at many different baselines using relatively small antennas.

This technique has been successfully employed in radio astronomy to obtain very high-resolution maps of radio sources in what is called "earth rotation synthesis" (Swenson and Mathur, 1968; Brouw, 1975; Hewish, 1965; Thompson, Moran and Swenson, 1986). The Very Large Array in Socorro, New Mexico is an example (Napier et al., 1983). Several versions of this technique have also been proposed for remote sensing from space. These include a proposal (Schanda, 
1976, 1979) in which the baseline is changed by making measurements at different frequencies, a concept (Mel'nik, 1972) involving a moving system with a single fixed baseline in which measurements must be made at several different time delays, and a modification which employs a scanning linear array which is rotated (C. Wiley, private communications). More recent variations include a configuration resembling a Mills cross in which multiple beams are formed with antenna elements along each arm of the cross (Milman, 1988); a variation resembling Mel'nik's configuration (Mel'nik, 1972) using contiguous parallel beams and matched filtering (Hughes Aircraft Co., private communications) and finally, a hybrid real and synthetic aperture system which uses real antennas to obtain spatial resolution in one dimension and aperture synthesis to obtain resolution in the other dimension (Le Vine et al., 1989; Swift et al., 1986).

Among the most important issues which must be resolved to determine the viability of aperture synthesis for remote sensing from space is the sensitivity $(\Delta T)$ which can be achieved with a particular configuration. Sensitivity is an especially critical issue for measurements from low earth orbit because the motion of the platform (about $7 \mathrm{~km} / \mathrm{s}$ ) limits the integration time available for imaging a particular scene. The problem is especially pernicious because the higher the spatial resolution required the less time is spent by the spacecraft over the scene. Other factors being constant, sensitivity depends on the actual (i.e. physical) collecting area of the antenna system employed. But the goal of aperture synthesis is to reduce the physical collecting area needed for a given spatial resolution. The sensitivity lost in this trade can, in many applications, be reclaimed by increasing the integration time-bandwidth product. However, this is not always possible for sensors in space because platform motion restricts the integration time, and requirements on the field-of-view and the problem of RFI restrict the bandwidth (Thompson and D'Addario, 1982). This trade-off between physical aperture and sensitivity was the motivation behind the hybrid real-and-synthetic aperture system mentioned above which was conceived as a means for achieving the sensitivity needed for soil moisture measurements from space (Le Vine et al., 1989; Ruf et al., 1988).

The purpose of this paper is to develop expressions for the sensitivity of remote sensing systems which use aperture synthesis. The objective is to develop basic equations general enough to be used to obtain the sensitivities of the several variations which have been proposed for sensors in space. The analysis is done in two parts: the first is a derivation of the sensitivity of the detector used to measure the signal from an individual pair of antennas (a coherent correlation receiver) and the second is the derivation of the sensitivity of the pixels in the final image. This is illustrated in Figure 1. On the left are shown two antennas viewing the scene. In aperture synthesis the antenna output voltages, $\mathrm{V}_{\mathrm{i}}$, are multiplied together and averaged. The middle panel of Figure 1 shows the schematic of a detector which makes this measure- 
ment. The output of this detector, $y_{i j}$, is a point in the Fourier transform space of the scene; and the scene itself is obtained by doing an inverse Fourier transform on the set of measurements $\left\{y_{\mathrm{ij}}\right\}$. This is done by the processor (far right) in Figure l. Many variations on this basic theme are possible. The variations may differ only in how the baselines are distributed in space or may involve subtle variations in how the signal processing is implemented. However, in each of the variations mentioned above, the correlator is a common element and the variations may all be cast in the form of Figure 1 with different processors. Hence, this paper will begin (Section II) with a discussion of the correlation receiver, deriving first the signal-to-noise ratio (SNR) and then the temperature sensitivity $(\Delta T)$ of the output. Then (Section III), an expression will be derived for the sensitivity in the final image, assuming that the processor is a discrete Fourier transform on the set of correlator outputs. It will be shown that in the most general case, the sensitivity may not be the same at each pixel in the image. Finally (Section IV), these results will be used to compare several of the variations of aperture synthesis mentioned above as potential candidates for remote sensing from space. The sensitivities of these configurations will also be compared with that obtained using a scanning, total power radiometer. The later result is obtained as a special case of the analysis done above.

\section{THE CORRELATION RECEIVER}

In aperture synthesis the coherent product (amplitude and phase) of the output voltages from a pair of antennas is measured for many pairs at different antenna spacings, and then an image is formed by processing this set. Ultimately, one is interested in the signal-to-noise ratio and sensitivity of the image. but as pointed out above, it is convenient to do the calculations in two steps, beginning with the receiver and then doing the calculations for the image. In this section, expressions are derived for the signal-to-noise ratio and the sensitivity of the correlation receiver.

\section{A. Signal-to-Noise Ratio (SNR)}

A schematic of the ideal correlation receiver is given as part of Figure 1 (middle). The receiver forms the coherent product of $\mathrm{X}_{\mathrm{i}}$ and $\mathrm{X}_{\mathrm{j}}$ which represents the voltages at the output terminals of the k-th pair of antennas. As part of the signal processing, these voltages would normally be amplified, filtered and mixed down to a convenient IF for further processing. The transfer functions, $H_{i}$ and $H_{j}$ represent the cumulative effect of this processing. $N_{i}$ and $N_{j}$ represent the equivalent noise in the circuit referred to the input and $\mathrm{H}_{\mathrm{LP}}$ is a low pass filter which represents the averaging (integration) which is done after the multiplication.

The input $X_{i}$ will be written in the form $X_{i}=X_{i}{ }^{\prime}+X_{i}$ where $X_{i}{ }^{\prime \prime}$ is the antenna output from a (perhaps hypotheti- 
cal) constant background against which changes are measured. The voltage $x_{i}$ is the change relative to this background which it is desired to detect. The signal $r_{i}$ at the input to the receiver (Figure l) is the sum of the antenna output and the system noise: $r_{i}=X_{i}{ }^{0}+N_{i}+x_{i}$.

Define the signal-to-noise ratio to be (e.g., Tiuri, 1964) the mean change in output due to the presence of the signal divided by the standard deviation of the output when the signal is zero (i.e., divided by the RMS noise). Using this definition, the SNR for the receiver output, $y_{i j}(r)$, is:

$$
S N R=\frac{\left\langle y_{i j}\left(X^{o}+N+x\right)>-<y_{i j}\left(X^{o}+N\right)>\right.}{\sqrt{<y_{i j}^{2}\left(X^{o}+N\right)>-<y_{i j}\left(X^{o}+N\right)>^{2}}}
$$

Expressions for the moments of $y_{i j}$ needed to evaluate Equation 1 can be obtained from Figure 1 using standard procedures (e.g., Appendix A). Since the signals $r_{j}(t)$ are the result of thermal noise, it is reasonable to assume that they are very broadband compared to the (bandpass) filters $H_{i}(v)$. In addition, assuming that $H_{L P}(v)$ is very narrow band compared to the $\mathrm{H}_{\mathrm{i}}(\mathrm{v})$, it can be shown (Appendix A) that:

$$
\begin{aligned}
& <\mathrm{y}_{\mathrm{ij}}(\mathrm{r})>=\mathrm{S}_{\mathrm{ij}}(\mathrm{v}) \mathrm{H}_{\mathrm{LP}}(0) \int_{-\infty}^{\infty} \mathrm{H}_{\mathrm{i}}(\mathrm{v}) \mathrm{H}_{\mathrm{j}}^{*}(\mathrm{v}) \mathrm{dv} \\
& \operatorname{Var}\left[y_{i j}(r)\right]=\left\langle y_{i j}{ }^{2}(r)>-<y_{i j}(r)\right\rangle^{2} \\
& =\left[S_{i j}{ }^{2}\left(v_{0}\right)+S_{i j}\left(v_{0}\right) S_{j j}\left(v_{0}\right)\right] \int_{-\infty}^{\infty} H_{i}{ }^{2}(v) H_{j}{ }^{2}(v) d v \int_{-\infty}^{\infty} H_{L P}{ }^{2}(v) d v
\end{aligned}
$$

where $S_{i j}(v)$ is the Fourier transform of the correlation function, $R_{i j}(\tau)=\left\langle r_{i}(t) r_{j}^{*}(t+\underline{\tau})\right\rangle$, and $v_{0}$ is the center frequency of the bandpass filters. The preceding expressions ignore "fringe washing," a problem which becomes important as the passband of $H_{i}(v)$ becomes large (Thompson and D'Addario, 1982). In employing Equations 2 it is convenient to make the following definitions: 


$$
\begin{aligned}
& B=\frac{\left[\int_{-\infty}^{\infty} H_{i}(v) H_{j}^{*}(v) d v\right]^{2}}{\int_{-\infty}^{\infty}\left[H_{i}(v) H_{j}(v)\right]^{2} d v} \\
& \bar{\tau}=\frac{H^{2}{ }_{L P}(O)}{\int_{-\infty}^{\infty} H^{2}{ }_{L P}(v) d v}
\end{aligned}
$$

where B is called the "noise bandwidth" of the receiver (Tiuri, 1964; Thompson et al., 1986) and $\tau$ is the equivalent integration time of the low pass filter (Kraus, 1966; Tiuri, 1964).

Now, substituting Equations 2 into Equation 1 and using the definitions in Equations 3, one obtains the following expression for the signal-to-noise ratio of the receiver:

$$
\text { S N R }=\frac{S_{i j}\left(v_{o}\right)}{\sqrt{S_{i j}{ }^{2}\left(v_{o}\right)+S_{i i}\left(v_{o}\right) S_{j j}\left(v_{o}\right)}} \sqrt{2 B T}
$$

In order to proceed further, the spectral densities, $S_{i j}(v)$, are needed. These are needed in two cases: a) when the input is the ambient background plus system noise $\left(r_{i}=X_{i}{ }^{0}+N_{i}\right)$, and b) when the input is the ambient background plus system noise plus the signal to be measured $\left(r_{i}=X_{i}^{0}+N_{i}+x_{i}\right)$. It will be assumed that the noise $N_{i}$ in the two channels of the radiometer are independent, and are independent of the ambient noise, $X_{i}{ }^{\circ}$. Then, in the first case $\left(r_{i}=X_{i}{ }^{0}+\right.$ $\mathbf{N}_{\mathrm{i}}$ ), one obtains the following correlation functions:

$$
\begin{aligned}
\mathrm{R}_{\mathrm{ij}}\left(\tau^{\prime}\right) & =\left\langle\left[\mathrm{X}_{\mathrm{i}}^{0}+\mathrm{N}_{\mathrm{i}}\right]\left[\mathrm{X}_{\mathrm{j}}^{0}+\mathrm{N}_{\mathrm{j}}\right]\right\rangle \\
& \left.=\quad<\mathrm{X}_{\mathrm{i}}^{0} \mathrm{X}_{\mathrm{j}}^{0}\right\rangle \\
\mathrm{R}_{\mathrm{ii}}(\tau) & =\left\langle\left[\mathrm{X}_{\mathrm{i}}^{0}+\mathrm{N}_{\mathrm{i}}\right]^{2}\right\rangle \\
& =\left\langle\mathrm{X}_{\mathrm{i}}^{\circ} \mathrm{X}_{\mathrm{i}}^{0}\right\rangle+\left\langle\mathrm{N}_{\mathrm{i}}^{2}\right\rangle
\end{aligned}
$$

where $\left\langle\mathrm{x}_{\mathrm{i}} \mathrm{x}_{\mathrm{j}}\right\rangle$ has been used as a shorthand notation for the correlation function $\left\langle\mathrm{x}_{\mathrm{i}}(\mathrm{t}) \mathrm{x}_{\mathrm{j}}{ }^{*}(\mathrm{t}+\tau)\right\rangle$ and the complex conjugate $\left(^{*}\right)$ has been dropped because the signals are real. If the noise and ambient signal in the two channels are the same, the results simplify and one may write: 


$$
\mathrm{R}_{\mathrm{ii}}\left(\tau^{\prime}\right)=\mathrm{R}_{\mathrm{jj}}(\tau)=\left\langle\mathrm{X}_{\mathrm{o}}^{2}\right\rangle+\left\langle\mathrm{N}_{\mathrm{o}}^{2}\right\rangle
$$

where $X_{i}{ }^{0}=X_{j}^{0}=X_{o}$ and $N_{i}=N_{j}=N_{o}$. In the second case $\left(r_{i}=X_{i}{ }^{0}+N_{i}+x_{i}\right)$, only the cross terms (i $\left.\neq j\right)$ are needed in Equation 4. One obtains:

$$
\begin{aligned}
& \mathrm{R}_{\mathrm{ij}}(\tau)=<\left[\mathrm{X}_{\mathrm{i}}^{\mathrm{o}}+\mathrm{N}_{\mathrm{i}}+\mathrm{X}_{\mathrm{i}}\right]\left[\mathrm{X}_{\mathrm{j}}^{\mathrm{o}}+\mathrm{N}_{\mathrm{j}}+\mathrm{x}_{\mathrm{j}}\right]> \\
& \left.=\left\langle\mathrm{X}_{\mathrm{i}}^{0} \mathrm{X}_{\mathrm{j}}^{\mathrm{o}}\right\rangle+<\mathrm{x}_{\mathrm{i}} \mathrm{x}_{\mathrm{j}}\right\rangle
\end{aligned}
$$

where it has been assumed that $\left\langle\mathrm{x}_{\mathrm{i}} \mathrm{X}_{\mathrm{j}}{ }^{\circ}\right\rangle=0$.

Now, the spectral densities $S_{i j}(v)$ needed in Equation 4 are obtained by Fourier transforming the $R_{\mathrm{ij}}(\tau)$ in Equations 6a and $6 \mathrm{~b}$. Denoting this Fourier transform by double brackets, $<<>>$, Equation 4 becomes:

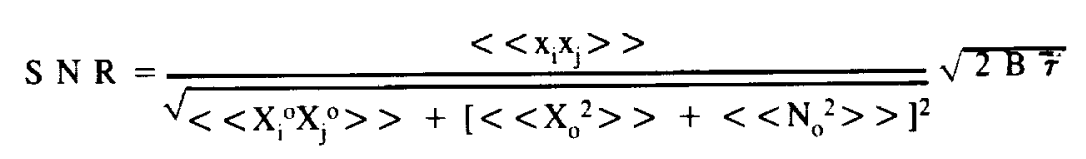

The Fourier transforms needed in Equation 7 can be derived following straightforward procedures (e.g., Appendix B; also see Fomalont and Wright, 1974). It turns out that the transforms are proportional to the spatial Fourier transform of the scene evaluated at a frequency $n=v_{0} L / c$ where $L$ is the distance between antennas. Since $X_{i}{ }^{\circ}$ is the output due to the ambient background, which by assumption is constant and the same for each antenna, the spectrum, $<<X_{i}{ }^{\circ}{ }_{j}{ }^{\circ}>>$ is zero except possibly at $\eta=0$. Thus, when $\eta \neq 0$, the SNR is:

$$
\text { S N R }=\frac{\left\langle<\mathrm{x}_{\mathrm{i}} \mathrm{x}_{\mathrm{j}}\right\rangle>}{\left.\left\langle<\mathrm{X}_{\mathrm{o}}{ }^{2}\right\rangle\right\rangle+\left\langle\left\langle\mathrm{N}_{\mathrm{o}}{ }^{2}\right\rangle\right\rangle} \sqrt{2 \mathrm{~B} \tau}
$$

When $\eta=0$ the correlation radiometer (Figure 1) is degenerate and it becomes a total power radiometer. In this case, the analysis follows as above except that $r_{i}=r_{j}$ and $H_{i}(v)=H_{j}(v)$. In this case, one obtains:

$$
\mathrm{SNR}=\frac{\left\langle<\mathrm{x}_{\mathrm{i}}^{2}\right\rangle>}{\left\langle<\mathrm{X}_{0}^{2}\right\rangle>+\left\langle\left\langle\mathrm{N}_{0}^{2}\right\rangle>\right.} \sqrt{\mathrm{B} \tau}
$$

which differs from Equation 8 only by a factor of 2 (Tiuri, 1964; Kraus, 1966).

\section{B. Sensitivity}

The sensitivity of the correlation receiver is obtained by setting the left-hand side of Equation 8 to unity (i.e., SNR $=1$ ) and solving for the fluctuation $\left\langle<\mathrm{x}_{\mathrm{i}} \mathrm{x}_{\mathrm{j}}>>\right.$ required to produce this SNR. However, before doing this it is 
convenient to change the notation in the preceding expressions to that commonly used in radio astronomy and remote sensing applications.

To proceed, notice that the Fourier transform, $\left\langle<\mathrm{x}_{\mathrm{i}} \mathrm{x}_{\mathrm{j}}\right\rangle>$, can be written in the following form (Appendix B; also see Swenson and Mathur, 1968):

$$
\left.<<x_{i} x_{j}\right\rangle>=\left(Z_{0} A_{e} / 2\right) \int_{-1}^{+1} \frac{P_{n}(q) B_{n}(\underline{q})}{\sqrt{1-q_{x}^{2}-q_{y}^{2}}} \exp (-j 2 \pi \underline{\eta} \cdot \underline{q}) d \underline{q}
$$

where $\underline{\eta}=\underline{\mathrm{L}}\left(v_{\mathrm{o}} / \mathrm{c}\right)$ and $\underline{\mathrm{L}}$ is the vector distance (i.e. "baseline") between the antennas, $Z_{0}=\sqrt{\mu_{\mathrm{o}} / \epsilon_{\mathrm{o}}}$ is the impedance of free space and $A_{e}$ is the effective receiving area of the antenna. (In the general case $\left\langle<x_{i} x_{j}\right\rangle>$ is a complex number; however, its complex conjugate is obtained trivially by interchanging antennas. Hence, one can always form a real signal to use in calculating the signal-to-noise ratio by averaging these two values: $\operatorname{Re}\left\langle\left\langle\mathrm{x}_{\mathrm{i}} \mathrm{x}_{\mathrm{j}}\right\rangle\right\rangle=\left[\left\langle\left\langle\mathrm{x}_{\mathrm{i}} \mathrm{x}_{\mathrm{j}}\right\rangle\right\rangle+\right.$ $<\left\langle\mathrm{x}_{\mathrm{j}} \mathrm{x}_{\mathrm{i}}\right\rangle>1 / 2$. This value will be used in Equation 8 to obtain the SNR. Additional discussion and an example of this point is given in Section IIIB.4.)

Before substituting into Equation 8, it is convenient to put Equation 10 into a more symmetric form by defining a normalized brightness temperature $B_{n}(q)$ as follows:

$$
B_{n}(\underline{q})=\frac{B(\underline{q})}{B_{v}(\underline{q})}=\left[\lambda^{2} / 2 k T_{B}\right] B(\underline{q})
$$

where $B_{v}(\underline{q})$ is the brightness of a constant thermal source of temperature $T_{B}$ (i.e., assuming that the Rayleigh-Jeans law applies to this source). With this definition, Equation 10 becomes:

$$
\left\langle\left\langle x_{1} x_{j}\right\rangle>=Z_{d,}\left[k T_{B} / \lambda^{2}\right]\right| A_{c} \int_{1}^{1} \frac{P_{n}(\underline{q}) B_{n}(\underline{q})}{\sqrt{1-q_{x}^{2}-q_{y}^{2}}} \exp (-j 2 \pi \underline{\eta} \cdot \underline{q}) d \underline{q}
$$

and defining $\theta(\underline{\eta})$ to be the following integral:

$$
\theta(\underline{\eta})=\operatorname{Re} \int_{-1}^{+1} \frac{P_{n}(\underline{q}) B_{n}(\underline{q})}{\sqrt{1-q_{x}^{2}-q_{y}^{2}}} \exp (-j 2 \pi \underline{\eta} \cdot \underline{q}) \cdot d \underline{q}
$$


one obtains:

$$
\operatorname{Re}\left\langle\left\langle\mathrm{x}_{\mathrm{i}} \mathrm{x}_{\mathrm{j}}\right\rangle>=\mathrm{Z}_{\mathrm{o}}\left[\mathrm{kT} \mathrm{T}_{\mathrm{B}} / \lambda^{2}\right] \mathrm{A}_{\mathrm{e}} \theta(\underline{\eta})\right.
$$

Notice that when $B_{n}(\underline{q})=1$ and $\underline{\eta}=0$ (i.e., the special case of a total power radiometer), then $\theta(\underline{\eta})$ reduces to the "solid angle" of the antenna used in the measurement (Kraus, 1966):

$$
\begin{aligned}
\Omega_{\mathrm{e}} & =\int_{\Omega} P_{\mathrm{n}}(\theta, \phi) \sin (\theta) \mathrm{d} \theta \mathrm{d} \phi \\
& =\int_{-1}^{+1} \frac{\mathrm{P}_{\mathrm{n}}(\underline{\mathrm{q}})}{\sqrt{1-\mathrm{q}_{\mathrm{x}}^{2}-\mathrm{q}_{y}^{2}}} \mathrm{dq} \\
& =\quad \lambda^{2} / \mathrm{A}_{\mathrm{e}}
\end{aligned}
$$

The transformation between the spherical coordinates $(\theta, \phi)$ and the direction cosines $\left(q_{x}, q_{y}\right)$ is straightforward ( Appendix D or Swenson and Mathur, 1968) and a derivation of Equation 14c can be found in Kraus (1966).

It is possible to obtain an explicit expression for $\left\langle\left\langle\mathrm{X}_{\mathrm{o}}{ }^{2}\right\rangle>\right.$, due to the ambient background, in terms of the notation introduced above. In particular, since $\left\langle<\mathrm{X}_{\mathrm{o}}{ }^{2}>>\right.$ is measured at $\eta=0$ and due to a constant $\left(\mathrm{B}_{\mathrm{n}}=1\right)$, one has $\left.<<X_{0}^{2}\right\rangle>=Z_{0}\left[k T_{X} / \lambda^{2}\right] A_{e}=Z_{0} k T_{X}$. Finally, assuming that the internal system noise is due to an equivalent source, but with temperature $\mathrm{T}_{\mathrm{N}}$, one can write: $\left\langle\left\langle\mathrm{N}_{\mathrm{o}}^{2}\right\rangle>=\mathrm{Z}_{0}, \mathrm{~T}_{\mathrm{N}}\right.$. Now using these expressions and Equations 13 and $14 \mathrm{c}$ in Equation 8, the SNR can be written:

$$
S N R=\frac{T_{B}}{T_{X}+T_{N}} \frac{\theta(\underline{\eta})}{\Omega_{e}} \sqrt{2 B \tau}
$$

Finally, the sensitivity of the correlation radiometer can be obtained by solving for the $T_{B}$ which yields a $S N R=1$. Calling this value $\Delta T$, one obtains:

$$
\Delta \mathrm{T}=\frac{\mathrm{T}_{\mathrm{sys}}}{\sqrt{2 \mathrm{~B} \tau}} \frac{\Omega_{e}}{\theta(\underline{\underline{\eta}})}
$$

where $T_{\mathrm{sys}}=\mathrm{T}_{\mathrm{x}}+\mathrm{T}_{\mathrm{N}}$ is the total "system" noise. Notice that the sensitivity, $\Delta \mathrm{T}$, depends on the antennas used in this measurement (i.e., on their solid angle, $\Omega_{\mathrm{e}}$ ), on the baseline between antennas $(\underline{\eta}$ ), and also on the structure of the source through $\theta(\eta)$. 


\section{Special Cases}

Equation 16 is a general expression and it will be used below to analyze several potential sensor systems which use aperture synthesis. However, before doing so several special cases will be considered to show that Equation 16 includes the standard results.

\section{Point Source}

First, consider the case when the scene is a point source. In this case, assuming that the point source is well within the main beam of the antenna so that $P_{n}(\underline{q}) / \sqrt{1-q_{x}{ }^{2}-q_{y}{ }^{2}} \cong 1$, one obtains $\theta(\underline{\eta})=\cos \left(2 \pi \underline{\eta} \cdot \underline{q}_{0}\right)$ where $\underline{q}_{0}$ is a unit vector pointing toward the source. Using this result, the sensitivity is:

$$
\Delta \mathrm{T}=\frac{\mathrm{T}_{\mathrm{sys}}}{\sqrt{2 \mathrm{~B} \tau}} \frac{\Omega_{\mathrm{e}}}{\overline{\cos \left(\phi_{0}\right)}} \quad \text { point source }
$$

where $\phi_{0}=2 \pi \underline{\eta}^{\bullet} \underline{\mathrm{q}}_{0}$. Notice that the singularity at $\cos \left(\phi_{0}\right)=0$ is only apparent because $\cos \left(\phi_{0}\right)=1$ near the main beam of the antenna, where it has been assumed that the point source is located. Equation 17 is identical to that derived by other methods (e.g., Tiuri, 1964; Kraus, 1966; chapter 7) except for the effect of the receiving antenna, $\Omega_{\mathrm{e}}$, which appears explicitly here. This factor doesn't appear (it is unity) if it is assumed that $T_{s y s}=T_{X}+T_{N}$ is the noise temperature of an equivalent point source (see below) as is done in the references cited above (e.g., Kraus, 1966).

\section{Total Pōer Radiometer}

As a second check, consider the case of a total power radiometer. The derivation follows as above but begins with Equation 9 for the SNR and uses $\underline{\eta}=0$ (zero baseline) in Equations 10,12, 13 and 15. The result is:

$$
\Delta \mathrm{T}=\frac{\mathrm{T}_{\text {sys }}}{\sqrt{\mathrm{B} \tau}} \frac{\Omega_{\mathrm{c}}}{\theta(0)} \quad \text { Total Power }
$$

In the special case of a uniform source which fills the beam of the antenna $(B=1)$, one has $\theta(0)=\Omega_{e}$ and the expression for the sensitivity becomes:

$$
\Delta \mathrm{T}=\frac{\mathrm{T}_{\mathrm{sys}}}{\sqrt{\mathrm{B} T}} \quad \text { Distributed Source }
$$

which is the classic result (Kraus, 1966; Ulaby et al., 1981). In this case the sensitivity is independent of the antenna 
aperture. However, notice that in the case of a point source (located very near the main beam of the antenna) one has $\theta(0)=1$ and the sensitivity is:

$$
\Delta T=\frac{T_{\text {sys }}}{\sqrt{B \bar{\tau}}} \Omega_{\mathrm{e}} \quad \text { Point Source }
$$

which depends on the antenna aperture. Clearly, the larger the antenna aperture $\left(\Omega_{e}=\lambda^{2} / A_{e}\right)$ the more sensitive the measurement will be to a point source.

\section{Noise Temperature Using Equivalent Point Source}

Finally, note that in each of the examples above, the equation for $\Delta T$ depends on the definition of $T_{B}$ and $T_{N}$. In particular, these could have been chosen to be the temperature of an equivalent point source rather than a distributed source (as was done above). Assuming they are due to a point source near the main beam of the antenna (i.e., at $\underline{\mathrm{q}}_{\mathrm{o}}=$ $0)$, then $\left\langle<\mathrm{X}_{\mathrm{o}}^{2}>>=\mathrm{Z}_{\mathrm{o}} \mathrm{k} \mathrm{T}_{\mathrm{X}} / \Omega_{\mathrm{e}}\right.$ and $\left.<<\mathrm{N}_{\mathrm{o}}{ }^{2}\right\rangle>=\mathrm{Z}_{\mathrm{o}} \mathrm{kT}_{\mathrm{N}} / \Omega_{\mathrm{e}}$. The derivation of $\Delta \mathrm{T}$ in this case follows as above, and the results can be obtained from Equations 15 through 18 by multiplying each equation by $1 / \Omega_{\mathrm{e}}$. For example, Equation 17 becomes $\Delta \mathrm{T}=\left[\mathrm{T}_{\mathrm{sys}} / \sqrt{\mathrm{B} \bar{\tau}}\right] / \cos \left(\phi_{\mathrm{o}}\right)$ and Equation $18 \mathrm{~b}$ becomes $\Delta \mathrm{T}=\left[\mathrm{T}_{\mathrm{sys}} / \sqrt{\mathrm{B} \tau}\right] / \Omega_{\mathrm{e}}$ but now Equation 18c is $\Delta \mathrm{T}=\mathrm{T}_{\mathrm{sys}} / \sqrt{\mathrm{B} \tau}$.

\section{SENSITIVITY OF THE IMAGE}

Equation 16 is the sensitivity of the output of a coherent correlation receiver (Figure 1) with an arbitrary, but fixed baseline $\eta$. In aperture synthesis, measurements are made at many baselines and then an image of the scene is constructed by taking a Fourier transform of these measurements (a discrete Fourier transform on the discrete set of measurements). In this section, an expression will be derived for the sensitivity in the final image when the processor in Figure $I$ is a discrete Fourier transform (DFT). This provides a basic formula from which several variations of aperture synthesis can be discussed (Section IV to follow).

For simplicity, assume that the measurements (receiver outputs, $y_{i j}$ ) are made at antenna baselines which can be mapped onto the coordinates of a Cartesian grid with uniform spacing, $d_{x}$ and $d_{y}$ along each axis, respectively. That is, assume that the measurements can be mapped onto the points $\left(\mathrm{md}_{\mathrm{x}}, \mathrm{n} \mathrm{d}_{\mathrm{y}}\right)$ where $\mathrm{m}=0,1,2 \ldots \mathrm{M}-\mathrm{I}$ and $\mathrm{n}=0$, $1,2 \ldots \mathrm{N}-1$. Also, let $\mathrm{I}_{\mathrm{mn}}$ be the intensity in the $\mathrm{mn}$-th pixel of the image. Then, with the processor being considered here, $I_{m n}$ is the two-dimensional discrete Fourier transform of the receiver outputs: $I_{m n}=$ DFT $\left[y_{m n}\right]$. The objective of 
this section is to derive an expression for the sensitivity of the $\mathrm{I}_{\mathrm{mn}}$ to changes in the scene.

\section{A. Signal-to-Noise Ratio (SNR)}

The initial step is to calculate the SNR and then the sensitivity is obtained by setting the SNR to unity. Adopting the same definition of signal-to-noise ratio for the image as was used for the receiver, one has:

$$
S N R=\frac{\left.<I_{m n}\left(X^{0}+N+x\right)>-<I_{m n}\left(X^{0}+N\right)\right\rangle}{\sqrt{\left.<I_{m n}^{2}\left(X^{0}+N\right)>-<I_{m n}\left(X^{0}+N\right)^{2}\right\rangle}}
$$

In this notation, $I_{m n}\left(X^{o}+N\right)=\operatorname{DFT}\left[y_{m n}\left(X^{0}+N\right)\right]$ is the intensity of the mn-th pixel in the image when the input is the ambient background $\left(X^{\circ}\right)$ plus system noise $\left.(N) .<I_{m n}\left(X^{0}+N\right)\right\rangle$ is a constant (d.c.) background noise in the image due to system noise and the ambient scene. The "signal" is the change in the scene relative to this ambient background and is due to $\mathrm{x}$.

Since the DFT is linear, the numerator in Equation 19 can be found immediately from Equations 2a, 5a and 6 b.

One obtains:

$$
<\mathrm{I}_{\mathrm{mnn}}\left(\mathrm{X}^{0}+\mathrm{N}+\mathrm{x}\right)>-<\mathrm{I}_{\mathrm{mn}}\left(\mathrm{X}^{0}+\mathrm{N}\right)>=\operatorname{DFT}\left\{<<\mathrm{x}_{\mathrm{n} 1} \mathrm{x}_{\mathrm{n}}>>\right\} \mathrm{H}_{\mathrm{LP}}(0) \int_{-\infty}^{\infty} H_{m}(v) H_{n}^{*}(v) d v
$$

In order to evaluate the denominator more information is needed about the output noise, $y_{1 m n}\left(X^{\prime \prime}+N\right)$. It is straightforward to show that $<y_{\operatorname{mn}}\left(X^{\circ}+N\right)>=0$ for all non-zero baselines (e.g. Equations 2a, 5a, and the discussion following Equation 7). In addition, it will be assumed that the $y_{m n}\left(X^{n}+N\right)$ are all independent and identically distributed. Then substituting into the definition of the discrete Fourier transform and using Equation 2b, one obtains:

$$
\begin{aligned}
& \left\langle\mathrm{I}_{\mathrm{mn}}^{2}\left(\mathrm{X}^{\prime \prime}+\mathrm{N}\right)\right\rangle-\left\langle\mathrm{I}_{\mathrm{mm}}\left(\mathrm{X}^{\prime \prime}+\mathrm{N}\right)^{2}\right\rangle=(\mathrm{I} / \mathrm{MN}) \mid\left\langle\mathrm{y}_{\mathrm{mmn}}{ }^{2}\left(\mathrm{X}^{\prime \prime}+\mathrm{N}\right)\right\rangle-\left\langle\mathrm{y}_{\mathrm{min}}\left(\mathrm{X}^{\prime \prime}+\mathrm{N}\right)\right\rangle^{2} \mathrm{I} \\
& =(1 / M N)\left[S^{2}{ }_{n n n}\left(v_{v}\right)-S_{n n}\left(v_{v}\right) S_{n m !}\left(v_{v}\right)\right] \int_{-\infty}^{\infty} H_{m !}(v) H_{n}^{*}(v) d v \int_{-\infty}^{\infty} H^{2} P(v) d v
\end{aligned}
$$

where $\mathrm{M}$ and $\mathrm{N}$ are the number of elements in each dimension of the discrete transform. Notice that the subscript "mn" has been kept in the equation above, although by assumption the result is independent of $m$ and $n$ (except possibly at $m$ 
$=0$ and $n=0$ where the receiver is degenerate and becomes a total power receiver). Now, using Equations $5 \mathrm{a}$ and $5 \mathrm{~b}$ and $6 \mathrm{a}$ in Equation $21 \mathrm{~b}$ and using the definitions given in Equations 3, one obtains:

$$
\text { S N R }=\frac{\text { DFT } \left.\left.\left\{<<\mathrm{x}_{\mathrm{m}} \mathrm{x}_{\mathrm{n}}\right\rangle\right\}\right\}}{\left.\left\langle<\mathrm{X}_{\mathrm{o}}{ }^{2}\right\rangle>+\left\langle<\mathrm{N}^{2}\right\rangle\right\rangle} \sqrt{2 \mathrm{MNB} \tau}
$$

Before calculating the sensitivity, it is convenient to rewrite Equation 22 in more conventional notation as was done above (Section IIB). Following the same procedure (i.e., defining a normalized brightness $B_{n}(\underline{q})$ as in Equation 11 and then using Equation 12), one obtains:

$$
\begin{aligned}
\operatorname{DFT}\left\{<<\mathrm{x}_{\mathrm{m}} \mathrm{x}_{\mathrm{n}}>>\right\} & =\mathrm{Z}_{\mathrm{\rho}}\left[\mathrm{k} \mathrm{T}_{\mathrm{B}}\right]\left[\mathrm{A}_{\mathrm{e}} \lambda^{2}\right] \int_{-1}^{+1} \frac{\mathrm{P}_{\mathrm{n}}(\underline{\mathrm{q}}) \mathrm{B}_{\mathrm{n}}(\underline{\mathrm{q}})}{\sqrt{1-\mathrm{q}_{\mathrm{x}}^{2}-\mathrm{q}_{\mathrm{y}}^{2}}} \mathrm{G}\left(\underline{q}_{\mathrm{mn}}-\underline{\mathrm{q}}\right) \mathrm{d} \underline{\mathrm{q}} \\
& =\mathrm{Z}_{\mathrm{o}}\left[\mathrm{k} \mathrm{T}_{\mathrm{B}}\right]\left[\mathrm{A}_{\mathrm{e}} / \lambda^{2}\right] \Theta(\mathrm{m}, \mathrm{n})
\end{aligned}
$$

where the following definitions have been made:

$$
\begin{aligned}
G\left(\underline{q}_{m n}-\underline{q}\right) & =\operatorname{DFT}\{w \exp (-j 2 \pi \underline{\eta} \cdot \underline{q})\} \\
\Theta(m, n) & =\int_{-1}^{+1} \frac{P_{n}(\underline{q}) B_{n}(\underline{q})}{\sqrt{1-q_{x}^{2}-q_{y}^{2}}} G\left(\underline{q}_{m n}-\underline{q}\right) d \underline{q}
\end{aligned}
$$

The factor, w, introduced in Equation 24a is an arbitrary "weight" which has been included in the DFT for completeness. It can be assigned arbitrarily to shape $G(\underline{q})$ or can be used to account for redundant measurements. The vector $g_{m, n}$ identifies each pixel in the image. Its components $\underline{q}_{m, n}=\left(q_{m}, q_{n}\right)$ in the special case of uniformly spaced baselines are $q_{m}$ $=m \lambda / d_{x} M$ and $q_{n}=n \lambda / d_{y} N$. (See Appendix F).

Now, assuming that $\left\langle<\mathrm{X}_{0}^{2}\right\rangle>=\mathrm{Z}_{\mathrm{o}} \mathrm{k} \mathrm{T}_{\mathrm{X}}$ and $\left\langle<\mathrm{N}^{2}>>=\mathrm{Z}_{\mathrm{o}} \mathrm{k} \mathrm{T}_{\mathrm{N}}\right.$, as done in Section IIB, and using Equation $23 b$, one obtains the following result for the signal-to-noise ratio:

$$
\text { S N R }=\frac{T_{B}}{T_{x}+T_{N}} \sqrt{2 M N B T} \frac{\Theta(m, n)}{\Omega_{e}}
$$

where $\Omega_{\mathrm{c}}$ is the "solid angle" of the antennas used in the measurements as defined in Equations 14. The sensitivity of the image is obtained by setting the SNR $=1$ and solving for the signal $T_{B}$ required to achieve $S N R=1$. Calling this signal 
$\Delta \mathrm{T}$, one obtains:

$$
\Delta T=\frac{T_{\text {sys }}}{\sqrt{2 \mathrm{~B} \tau}} \frac{1}{\sqrt{\mathrm{MN}}} \frac{\Omega_{\mathrm{e}}}{\Theta(\mathrm{m}, \mathrm{n})}
$$

Notice that since $\theta(m, n)$ depends on $m$ and $n$, the SNR and sensitivity, $\Delta T$, may be different for each pixel. However, while this is true in general, it is not a problem for the special cases most commonly encountered in practice. Examples will be given in the special cases to be discussed below.

\section{B. Special Cases}

In the case of uniformly spaced measurements, $G(\underline{q})$ has the form of the array factor encountered in the analysis of uniform, linear antenna arrays (Appendix F; also, see Jordan and Balmain, 1968). In applications such as remote sensing from space, the number of individual antennas in the synthesis array is likely to be large (e.g., Le Vine et al., 1989), in which case $G\left(\underline{q}_{m n}-\underline{q}\right)$ will be a rapidly varying function in comparison to $P_{n}(\underline{q})$ with its support near $\underline{q}_{m n}$. Hence, near the center of the image where $\left|\underline{q}_{m n}\right|<<1$ one obtains:

$$
\theta(m, n)=\int_{-1}^{+1} B_{n}(\underline{q}) G\left(\underline{q}_{m, n}-\underline{q}\right) d \underline{q}
$$

This is just the expression for the power received by an antenna with the power pattern, G(q), when viewing the source $B_{n}$ (q) (e.g., Kraus, 1966; Collin and Zucker, 1969). Hence, close to the center of the image, $\Theta(m, n$ ) will depend on $(m, n)$ only to the extent that the scene, $B_{n}(q)$, changes from pixel to pixel.

Before proceeding, it is convenient to define a solid angle, $\Omega_{\mathrm{syn}}$ and an equivalent area, $A_{\mathrm{syn}}$, for the "synthesized beam." In light of the discussion above (Equations 24 and 27) and in analogy with Equations 14, the following definitions are made:

$$
\begin{aligned}
\mathbf{\Omega}_{\mathrm{syn}} & =\int_{1}^{1} \frac{\mathbf{P}_{\mathrm{n}}(\underline{q})}{\sqrt{1-\mathrm{q}_{\mathrm{x}}{ }^{2}-\mathrm{q}_{\mathrm{y}}{ }^{2}}} \mathbf{G}(\underline{q}) \mathrm{dq} \\
& =\lambda^{2} / A_{\mathrm{yyn}}
\end{aligned}
$$


With these definitions, Equation 26 can be written in a particularly appealing form in a few important, special cases as discussed below.

\section{1) Distributed Source}

First, consider the case of a distributed source which is constant over the dimension of one pixel. Then $\Theta(\mathrm{m}, \mathrm{n}) \cong$ $\Omega_{\text {syn }}=\lambda^{2} / A_{\text {syn }}$ and Equation 26 becomes:

$$
\Delta T=\frac{T_{\text {sys }}}{\sqrt{2 \mathrm{~B} T}} \frac{\mathrm{I}}{\sqrt{\mathrm{MN}}} \frac{\mathrm{A}_{\mathrm{syn}}}{\mathrm{A}_{\mathrm{e}}}
$$

This is the form commonly found in radio astronomy applications (Napier et al., 1983; Thompson et al., 1986).

\section{2) Point Source}

Next, consider the case of a point source located in the main beam at $\underline{q}_{m . n}$. In this case, one has $\theta(m, n) \cong 1$ and Equation 26 becomes:

$$
\Delta T=\frac{T_{s y s}}{\sqrt{2 B \tau}} \frac{1}{\sqrt{M N}} \Omega_{e}
$$

\section{3) Zero Redundancy}

Additional insight can be obtained by examining the factor, MN, in Equations 29. Consider $\mathrm{n}$ antennas and suppose that measurements are made at all possible antenna pairs. There are $n(n-1) / 2$ different pairs. If no pairs have the same baseline (and the baselines are uniformly spaced), then this arrangement is referred to as a "zero redundancy" array. In practice, more than $n$ antennas would be required to achieve the $n(n-1) / 2$ baselines because zero redundancy arrays have been found in only a few cases (e.g., Moffet, 1968). However, it is convenient to assume a zero redundancy array in order to obtain an expression for $\Delta \mathrm{T}$ which explicitly shows its dependence on the number of individual antennas in the synthesis array. Hence, let $M N=n(n-1) / 2$ and, since $n$ is likely to be large for any practical application in remote sensing from space, one may further simplify by writing $M N \cong n^{2} / 2$. Thus, in the case of a zero redundancy array of $n$ antennas, Equations 29 can be written:

$$
\Delta T= \begin{cases}\frac{T_{\mathrm{sys}}}{\sqrt{\mathrm{B} \tau}} \frac{A_{\mathrm{syn}}}{\mathrm{n} A_{\mathrm{c}}} & \text { distributed source } \\ \frac{T_{\mathrm{sys}}}{\sqrt{\mathrm{B} \tau}} \frac{\Omega_{\mathrm{c}}}{\mathrm{n}} & \text { point source }\end{cases}
$$


Equation 30a is the expression commonly quoted in the literature on applications to radio astronomy (e.g., Napier et al., 1983). Clearly, in either case (point source or distributed source), reducing the product, $n A_{e}$, worsens the sensitivity. Hence, in applications to remote sensing from space there is a trade to be made between thinning the array to reduce the size and weight in orbit, and obtaining the best possible sensitivity.

\section{4) Hermitean Symmetry}

More data are available to create the image than the $M N=n(n-1) / 2$ points discussed above. In particular, the signal $<<x_{m} x_{n}>>$ at any baseline $(m, n)$ is Hermitean: $\left.\left\langle<x_{m} x_{n}\right\rangle=\left\langle<x_{-n} x_{-m}\right\rangle\right\rangle^{*}$ which can be seen from Equation 10 using the vector baseline $\underline{L}=m d_{x} x+n d_{y} y$. Hence, given measurements at $\mathrm{MN}$ baselines, the total

number of points available for each dimension in the DFT are $\hat{M}=2 M+1$ and $\hat{N}=2 N+1$ which are made up of the $\mathrm{m}=1,2,3 \ldots \mathrm{M}$ and $\mathrm{n}=1,2,3 \ldots \mathrm{N}$ actual measurements, the complex conjugate of these measurements and the measurement at zero baseline. Using all of these points in the DFT creates an image which is real (real values of brightness temperature). Using all of them results in a gain function $\mathrm{G}(\underline{\mathrm{q}})$ which is real and symmetric. However, since the conjugate points do not represent physically different measurements, the noise in these data is not independent. Hence, including them in the processor does not improve the sensitivity. These additional data points improve the resolution but not the sensitivity.

\section{SELECTED CONFIGURATIONS}

Equation 26 and its special cases (Equations 29 and 30) provide the basis for the evaluation of several different variations of aperture synthesis which have been proposed for remote sensing from space. Expressions for the sensitivity of the image will be derived in this section for the following configurations: a) a scanning real aperture antenna using a total power receiver (a special case of the correlation receiver); b) a representative synthetic aperture radiometer in which the antennas are arranged along the arms of " $T$;" c) a hybrid real/synthetic aperture sensor (called "ESTAR") which has been proposed to make soil moisture measurements from space as part of NASA's Earth Observing System (Eos); and d) a variation in which individual antennas are arranged in a "cross" or " $\mathrm{T}$ " but instead of doing traditional aperture synthesis, the antennas are combined to form a linear array with multiple beams and the output from orthogonal beams are correlated to obtain resolution in two dimensions like a multiple-beam Mills cross (e.g., Milman, 1988). Expressions for 
the sensitivity of each variation will be obtained in this section and then the sensors will be compared for a hypothetical remote sensing application in the section to follow.

\section{A. Scanning, Total Power Radiometer}

The most common way a microwave radiometer is used to form an image is to scan the beam across the scene, spending $\tau$ seconds at each spot. Systems with both electrical and mechanical scanning have been used for microwave remote sensing from space (e.g., ESMR, SMMR, SSM/I). In such a system, each pixel is the result of a separate measurement and the sensitivity is just that of the receiver output. The receiver sensitivity can be obtained using the analysis in the preceding sections in the special case of zero baseline. Using the SNR as given in Equation 9, and following the same arguments which were used to obtain the sensitivity of the correlation receiver (Equations 10 through 17), one obtains the following expression for the sensitivity of the total power radiometer viewing an extended source:

$$
\Delta \mathrm{T}=\frac{\mathrm{T}_{\mathrm{sys}}}{\sqrt{\mathrm{B} \tau}}
$$

This is the conventional result for a total power receiver (e.g., Kraus, 1966) and as mentioned above, it applies to each pixel in the final image.

Notice, that the sensitivity in this case (distributed source) does not depend on the antenna aperture. Also notice that Equation 31 differs from that obtained with aperture synthesis (Equation 30a) by the factor $A_{s y n} / n A_{c}$ which is the ratio of the effective area of the synthesized beam to the effective area of the antennas actually used in the array. Since $n A_{e}$ $<\mathrm{A}_{\text {syn }}$ in any practical application, the synthesized beam will have a poorer sensitivity than a real antenna of equal effective area if all other factors (i.e., B and $\tau$ ) are the same. However, as will be discussed below, the integration time, $\tau$, available per pixel can be very different for the real aperture and synthetic aperture sensors. In particular, because the synthetic aperture sensor doesn't have to scan, the integration time can be much longer.

\section{B. Synthetic Aperture Array}

A synthetic aperture radiometer with a prescribed spatial resolution can be implemented with individual antennas in many different configurations. The only requirement is that the antenna positions provide the proper sampling in the spatial frequency domain. For example, the antennas could be arranged along the circumference of a circle, along the arms of a "cross," along the arms of a "Y" or "T," or even in a "random" array, and in each case obtain a synthesized 
beam with roughly the same resolution. In designing an array for aperture synthesis, one has a great deal of flexibility to configure the system to accommodate the platform and to provide holes to avoid blocking the fields of view of other sensors. The sensitivity of a representative example will be discussed in this section to provide a reference.

The example to be treated explicitly consists of identical antennas uniformly spaced along the arms of a "T."' This is a convenient example to analyze because the independent baselines which can be formed in such an arrangement map onto a Cartesian grid $(x, y)$ where $x$ is an integer multiple of the distance between antennas along one arm of the " $T$ ",

(e.g., the top) and where $y$ is an integer multiple of the distance between antennas along the other arm. If $N_{x}$ and $N_{y}$ are the number of antennas along each of the arms, then the number of independent baselines is the product $N_{x} N_{y}$, and the sensitivity obtained with this configuration is obtained from Equation $29 a$ with $M N=N_{x} N_{y}$ :

$$
\Delta T=\frac{T_{s y s}}{\sqrt{2 B \tau}} \frac{1}{\sqrt{N_{x} N_{y}}} \frac{A_{s y n}}{A_{e}}
$$

For the sake of simplicity, assume that each arm of the " $T$ " is of equal length, $L$, and contains the same number of identical antennas, each with dimension $\mathrm{W}$ (i.e., square patches $\mathrm{W}$ meters on a side). Then, to a first approximation $\mathrm{A}_{\text {syn }}=$ $L^{2}$ and $A_{e}=W^{2}$ and letting $N_{x}=N_{y}=N$, the sensitivity becomes:

$$
\begin{aligned}
\Delta \mathrm{T} & =\frac{\mathrm{T}_{\mathrm{sys}}}{\sqrt{2 \mathrm{~B} \tau^{\prime}}} \frac{\mathrm{L}^{2}}{\mathrm{~N} \mathrm{W^{2 }}} \\
& =\frac{\mathrm{T}_{\mathrm{sys}}}{\sqrt{\mathrm{B} \tau}} \frac{\mathrm{L}}{\sqrt{2} \mathrm{~W}}
\end{aligned}
$$

where the last expression is obtained using the upper limit, $N=L / W$, to estimate the number of antennas.

It may be possible to improve the sensitivity in some remote sensing applications by averaging pixels. In particular, the synthetic aperture radiometer forms an image of the entire field of view during each integration period ( $\tau$ seconds); and because the platform (e.g., a satellite in low earth orbit) is moving, the next image will be shifted slightly in the direction of motion. If the integration time is chosen so that this shift is just one pixel, then each pixel will appear in several successive images ( $\mathrm{N}$ times in the example being discussed here). Each view will not be at the same incidence angle, but if this difference will not degrade the data, then it is possible to use these multiple looks to further reduce the 
noise. Assuming that each look is independent, one obtains an improvement of $1 / \sqrt{N}$, and using $N=L / W$ the sensitivity (Equation 33) becomes:

$$
\Delta \mathrm{T}=\frac{\mathrm{T}_{\mathrm{sys}}}{\sqrt{\mathrm{B} \tau}} \sqrt{\frac{\mathrm{L}}{2 \mathrm{~W}}}
$$

At first glance, the sensitivity of this synthetic aperture radiometer, even in the optimum form given in Equation 34 , is poorer than is obtained with a scanning, total power radiometer (Equation 31); however, this is misleading because the integration time available to form an image in the two cases is very different. In particular, in order for the real aperture radiometer to form a map comparable to that obtained with the synthetic aperture radiometer (i.e., a map consisting of $\mathrm{N}$ $\times N$ contiguous pixels), it must complete a scan in $t^{\prime} / N$ seconds where $t^{\prime}$ is the time the spacecraft is over one pixel. The integration time available for aperture synthesis (Equation 34) is $t^{\prime}$ and the time available for the scanning, total power radiometer (Equation 31 ) is $t^{\prime} / N$. Using $t^{\prime}=1 / N=W / L$ in Equation 31 yields a result which differs from Equation 34 by only $1 / \sqrt{2}$. Hence, in this case, synthetic and real aperture sensors with comparable system temperatures and bandwidths provide comparable radiometric sensitivity.

\section{Hybrid Real and Synthetic Aperture Array (ESTAR)}

The sensitivity obtained using aperture synthesis (Equation 30a) depends inversely on the actual collecting area $\left(\mathrm{nA}_{\mathrm{e}}\right)$ employed in the array and also inversely on the time-bandwidth product of the measurement. In remote sensing applications in space, the integration time is limited by the platform motion (about $7 \mathrm{~km} / \mathrm{sec}$ in low earth orbit) and the bandwidth is limited by RFI and requirements on the field of view of the instrument (Thompson and D'Addario, 1982).

Hence, in general, a trade has to be made between the sensitivity which must be achieved for a given measurement to be useful and the reduction in antenna hardware (i.e., $\mathrm{nA}_{\mathrm{e}}$ ) which can be obtained. ESTAR is one such compromise which has been proposed to make measurements of surface soil moisture practical from space (Murphy et al., 1987; Le Vine et al., 1989). The idea is to increase sensitivity by doing aperture synthesis in only one dimension. ESTAR achieves spatial resolution in the along-track dimension by employing long stick antennas (real apertures) aligned parallel to the motion of the spacecraft and achieves resolution in the other (cross-track) dimension by means of aperture synthesis using pairs of the stick antennas. Using aperture synthesis substantially reduces the number of stick antennas required (Le Vine et al., 1989; Swift et al., 1986).

The sensitivity of this configuration is obtained directly from Equation $30 \mathrm{a}$ where $A_{e}$ is the area of the stick anten- 
nas and $n$ is the number of sticks employed. Letting the sticks be $L$ meters long and $\mathrm{W}$ meters wide, and using the expressions $A_{e}=W L$ and $A_{\text {syn }}=L^{2}$ to approximate $A_{e}$ and $A_{\text {syn }}$, one obtains:

$$
\Delta T=\frac{T_{\text {sys }}}{\sqrt{B T}} \frac{L}{n W}
$$

This is the sensitivity of each pixel in the image formed by this hybrid sensor. The ratio, $\mathrm{L} /(\mathrm{nW})$, is the "fill factor" of the array: it is the fraction of the total available area which is occupied with receiving antennas. The goal is to reduce the fill factor as much as possible to save weight while at the same time achieving the required sensitivity. If one assumes that $\tau$ is fixed by the velocity of the platform and B is limited by fringe washing, then the ratio $\mathrm{L} / \mathrm{nW}$ determines the amount of thinning that can be achieved and still meet the sensitivity requirement of the measurement. In the application to soil moisture, this array could be more than 80 percent empty space (e.g., Le Vine et at., 1989).

\section{Correlation Cross Antenna}

Another sensor system to which the formulas above apply is the variation of the Mills cross described recently by Milman (1988). In this system, two linear arrays are arranged orthogonally in the form of a "cross" or "T."

The antenna elements in each arm are used to form a collection of contiguous beams with resolution in the dimension perpendicular to that arm. The intersection from a pair of beams (one from each arm) determines the resolution "cell" and the signal from this cell is detected by correlating the output from this pair of beams. For example, let $\mathrm{X}_{\mathrm{i}}$ be the $\mathrm{i}$-th beam formed with an array along the $x$-axis and $Y_{j}$ be the $j$-th beam formed with an array along the other arm. Then, in the proposed system, the signal from the intersection of these two beams is detected by forming the product $\left\langle\mathrm{X}_{\mathrm{i}}(\mathrm{t}) \mathrm{Y}_{\mathrm{j}}{ }^{*}(\mathrm{t})\right\rangle$ in the processor. Radiation from the entire scene can be mapped using contiguous beams and forming the product of all pairs $(i, j)$.

Although this system could be implemented with a collection of beam forming networks and correlators (Milman, 1988), it can also be implemented as a special case of aperture synthesis in which the individual antennas in the synthesis array are aligned along the arms of the cross. To see this, first note that the correlator required to form the product is the same as indicated in Figure 1 (correlation receiver) with the two arms $\left(x_{i}\right)$ representing the signal from two beams. Consequently, the result of multiplying the two beams together is the same as given in Equation 2a, but with $S_{i, j}(v)$ representing the spectrum formed from the product of two beams. It is the Fourjer transform of the correlation function $<\mathrm{X}_{\mathrm{i}}(\mathrm{t}) \mathrm{Y}_{\mathrm{j}}(\mathrm{t}+\tau)$. Now, to compare with aperture synthesis, assume that each arm consists of $2 \mathrm{~N}+1$ identical, uniformly 
spaced elements whose output voltage (in the frequency domain) is $V_{n}(v)$ where $n=0, \pm 1, \pm 2, \pm 3 \ldots \pm N$. Then the i-th beam is formed by adding an appropriate phase to each element in the array (e.g., Jordan and Balmain, 1968). In the frequency domain this can be written:

$$
\begin{aligned}
X_{i}(v) & =\quad \text { output of the i-th beam } \\
& =\sum_{n=-N}^{N} v_{x n}(v) \exp \left(j n k d q_{x i}\right)
\end{aligned}
$$

where $k=2 \pi v / c, d$ is the spacing between elements and $q_{x i}$ is the direction cosine of the vector in the direction of the main lobe (main beam) of the $i$-th beam measured relative to the axis of the array $\left(-1<q_{x i}<+1\right.$ and $q_{x i}=0$ at broadside). Now, the correlation function for two beams can be written in terms of its frequency domain representation as follows:

$$
\left.\left\langle\mathrm{X}_{i}(\mathrm{t}+\tau) \mathrm{Y}_{\mathrm{j}}^{*}(\mathrm{t})\right\rangle=\int_{-\infty}^{\infty}<\mathrm{X}_{\mathrm{i}}(\mathrm{v}) \mathrm{Y}_{\mathrm{j}}^{*}(\mathrm{v})\right\rangle \exp (-\mathrm{j} 2 \pi \mathrm{v} \tau) \mathrm{dv}
$$

and using Equations 36 and the assumption that $\left\langle\mathrm{V}_{\mathrm{xn}}(\mathrm{v}) \mathrm{V}_{\mathrm{ym}}{ }^{*}\left(\mathrm{v}^{\prime}\right)\right\rangle=\left\langle\mathrm{V}_{\mathrm{xn}}(\mathrm{v}) \mathrm{V}_{\mathrm{ym}}{ }^{*}(\mathrm{v})\right\rangle \delta\left(\mathrm{v}-\mathrm{v}^{\prime}\right)$ and re-ordering the sum on $n$, one has:

$$
\left.\left\langle X_{i}(v) Y_{j}^{*}(v)\right\rangle=\sum_{m=-N}^{N} \sum_{n=-N}^{N}<v_{x n}(v) V_{y m}{ }^{*}(v)\right\rangle \exp \left[-j k d\left(n q_{x i}+m_{x i}\right)\right]
$$

The average $\left\langle\mathrm{x}_{\mathrm{i}}(v) \mathrm{X}_{\mathrm{j}}(\mathrm{v})\right\rangle$ is the spectrum $S_{\mathrm{ij}}(v)$ in Equation 2a when the inputs to the correlator (Figure 1) are the beams formed by the two arms of the cross. Notice, that it has the form of a discrete Fourier transform of the average $\left\langle V_{x n}(v) V_{y m}(v)\right\rangle$, which in turn, is the spectrum $S_{i j}(v)$ in Equation 2a when the inputs to the correlation receiver are the signals from the individual antennas in each arm. Hence, Equation $37 \mathrm{~b}$ represents the signal processor required to form an image when employing aperture synthesis. Thus, the process of forming and correlating a pair of beams is just an alternative way of implementing the "processor" in Figure 1 required in aperture synthesis. As long as the two arms of the cross intersect (i.e. have an element in common) then the sum in Equation $37 \mathrm{~b}$ involves all possible pairs of antennas and the image formed is identical to that obtained with aperture synthesis when employing the same configuration of elemental antennas. 
It follows that Equations 16 and 26 for the sensitivity of a synthetic aperture imaging system also apply to this configuration. In the case when the scene is extended, Equation 26 reduces to Equation $30 \mathrm{a}$ and one has:

$$
\Delta T=\frac{T_{\text {sys }}}{\sqrt{2 \mathrm{~B} T}} \frac{1}{\sqrt{\mathrm{MN}}} \frac{\mathrm{A}_{\mathrm{syn}}}{\mathrm{A}_{\mathrm{e}}}
$$

This applies to the cross configuration being considered here where $T_{\text {sys }}$, B and $\tau$ are the system noise temperature, bandwidth and integration time of the correlator used for each pair of beams; $A_{e}$ is the collecting area of each elemental antenna; $A_{\mathrm{syn}}$ is the effective area of the beam formed by each arm of the cross; and $\mathrm{MN}$ is the number of independent pairs of individual antennas in the cross.

In order to facilitate comparison with the ESTAR configuration described above, assume arms of length, L, and width, $\mathrm{W}$, and suppose that there are $\mathrm{N}=\mathrm{L} / \mathrm{W}$ antennas in each arm. Then, $\mathrm{A}_{\mathrm{syn}}=\mathrm{L}^{2}$ and $\mathrm{A}_{\mathrm{e}}=\mathrm{W}^{2}$, and there are $\mathrm{N}^{2} / 2$ independent antenna pairs in this array. (Only the baselines formed between one arm and half of the other arm of a cross, “+”, are independent.) Thus, with $\mathrm{MN}=\mathrm{N}^{2} / 2=\mathrm{L}^{2 /\left(2 \mathrm{~W}^{2}\right)}$ Equation 38 can be written:

$$
\Delta \mathrm{T}=\frac{\mathrm{T}_{\mathrm{sys}}}{\sqrt{\mathrm{B} \tau}} \frac{\mathrm{L}}{\mathrm{W}}
$$

However, it may be possible to obtain better sensitivity by averaging the pixels in successive images as done above (Section IVB). This can be done on a moving platform by adjusting the integration time so that the shift due to platform motion during the integration is just one pixel. Then each pixel will be appear in several successive images ( $\mathrm{N}$ times). Assuming that each look is independent and that the difference in incidence angle will not degrade the data, one obtains an improvement of $1 / \sqrt{\mathrm{N}}=\sqrt{\mathrm{W} / \mathrm{L}}$ and Equation 35 becomes:

$$
\Delta \mathrm{T}=\frac{\mathrm{T}_{\mathrm{sys}}}{\sqrt{\mathrm{B} \tau}} \frac{\mathrm{L}}{\mathrm{W}}
$$

In summary, it should be clear from this discussion that one could implement this system either with a collection of beam forming networks and correlation receivers, or by forming all the $\mathrm{n}$ correlation pairs first and then doing the beam forming (a DFT). In the latter case the system is clearly a conventional form of aperture synthesis.

\section{COMPARISON}

In order to compare the performance of the several configurations described above, consider as a specific example the problem of measuring soil moisture from space. Imagine a sensor in polar orbit as part of the proposed "Earth 
Observing System"' (Butler et al., 1988). Preliminary studies call for an instrument at L-band (1.4 GHz) with a spatial resolution of about $10 \mathrm{~km}$ (Murphy et al., 1987; Le Vine et al., 1989). At the orbit proposed for the Eos (824 km) this means an antenna aperture on the order of $20 \mathrm{~m}$ on a side, clearly a candidate for aperture synthesis in some form. It will be assumed that the platform is moving at $7 \mathrm{~km} / \mathrm{s}$ and that a swath of $1000 \mathrm{~km}$ (global coverage every 3 days) is required. The integration time will be chosen to be the time available per resolution cell. That is $\tau=10 \mathrm{~km} \div 7 \mathrm{~km} / \mathrm{sec}$ for the synthetic aperture sensor and $\tau=(10 \mathrm{~km} \div 7 \mathrm{~km} / \mathrm{sec}) \div 100$ for the scanning, real aperture radiometer (where 100 is the number of resolution cells per scan line). A bandwidth of $10 \mathrm{MHz}$ will be assumed for the synthesis arrays (a reasonable value for which fringe washing should not be a problem and digital processing can be readily implemented) and a bandwidth of $30 \mathrm{MHz}$ has been assumed for the total power radiometer (this is the full bandwidth available at the $1.4 \mathrm{GHz}$ radio astronomy band and, although a larger bandwidth is technically feasible, a system with a larger bandwidth is likely to encounter severe RFI problems at L-band). A system noise temperature $T_{s y s}=500 \mathrm{~K}$ will be used for all sensors. This is a conservative estimate, and lower system temperatures likely can be achieved.

Table $I$ is a comparison of the sensitivity of each of the candidate configurations discussed above. The first row in the table gives the general theoretical expression for the sensitivity in the image using the formulas derived above for the case of a distributed target. The second row gives the sensitivity for the specific configuration under consideration. The synthetic aperture configurations being considered are: 1) a representative configuration with individual antenna elements uniformly spaced along the arms of a "T"; 2) the "cross" configuration; and 3) the hybrid synthetic and real aperture sensor, "ESTAR." Expressions for the sensitivity of the former two configurations are given both with and without additional pixel averaging. The total power radiometer is assumed to have an antenna 20 meters on a side and the length of the array in each of the synthetic aperture configurations will be assumed also to be $20 \mathrm{~m}$ to achieve approximately the same resolution with all of the sensor configurations. The width of the array has been assumed to be about one-half a wavelength $(0.1 \mathrm{~m})$ and it is assumed that each elemental antenna in the array is $\mathrm{W}=0.1 \mathrm{~m}$ on a side. This permits a minimum spacing between antennas of one-half a wavelength in all the synthesis arrays. An improvement in sensitivity could be achieved by using bigger elemental antennas (larger $\mathrm{W}$ ); however, when $\mathrm{W}>\lambda$ (approximately) the grating lobes are no longer in imaginary space. The number of antennas in each array is L/W except for ESTAR. In the ESTAR configuration, aperture synthesis is done only in one dimension, and in this configuration the number of sticks has been chosen to be the number needed in a minimum redundancy array to achieve spacings which are an integer multiple of $\lambda / 2$. In practice, more antennas would be needed, but no additional independent spacings would be achieved. The cross is 
assumed to be in the form of "+". Thus, one only uses N/2 elements in one of the arms which explains the factor of 2 in the formulas appearing in the Table. The "+" will also affect resolution somewhat, but this is being ignored. The cross could be implemented in the form of a "T" in which case, the formulas are exactly the same as for the synthetic aperture array having the same form.

The third row in Table I gives a numerical value for the sensitivity obtained using the parameters shown at the bottom of the Table. Notice, in particular, that when pixel averages are employed in the "cross" and " $T$ " configurations, the sensitivities of both the real aperture and synthetic aperture are about the same. However, without pixel averaging, only the "ESTAR" configuration offers a sensitivity, $\Delta \mathrm{T}$, close to that achieved with a real aperture, scanning radiometer.

\section{CONCLUSIONS}

General expressions have been derived for the sensitivity of a radiometer which uses aperture synthesis to form an image. Among the interesting conclusions is that the sensitivity of the individual correlation receiver, of which such a radiometer is comprised, depends on both the baseline (distance between antennas) and on the nature of the scene (e.g.. point source compared to distributed source). The same is true of the image formed with aperture synthesis from a collection of such receivers. The sensitivity can be different for different pixels in the image and in addition, can depend on the nature of the source. This result is different than in the case of a conventional imaging radiometer system (i.e., a scanning total power radiometer) where a single receiver and a single antenna are used to form an image. In this case, the sensitivity can be made independent of the antenna and scene by an appropriate definition of the system noise temperature (e.g., see Equation 18).

In the situations likely to be encountered in microwave remote sensing of the earth from space, the expressions for the sensitivity given above can be simplified (e.g., assuming a minimum redundancy array with a large number of elements and a distributed source). In this case, the sensitivity obtained with aperture synthesis is proportional to that obtained with a total power radiometer of the same system temperature, bandwidth and integration time (i.e., $\mathrm{T}_{\text {sys }} / \sqrt{\mathrm{B} \tau}$ ) The proportionality constant is the "fill" factor, $A_{\text {syn }} / n A_{i c}$, which is the ratio of the effective area of the synthesized antenna to the actual collecting area employed in the array.

The advantage of aperture synthesis is that it can achieve spatial resolutions equivalent to total power radiometers with large effective collecting areas (i.e., equal to $A_{\text {syn }}$ ), but using small antennas $\left(A_{c}\right)$. The reduction in sensitivity that 
this entails can be restored because the synthetic aperture system does not need to scan and it collects energy from many independent antenna pairs. The comparisons given in Table I indicate that very comparable sensitivity can be obtained with several synthetic aperture systems which have very substantial reductions in collecting area. Although the comparison applies specifically to the special case of soil moisture measurements from space, similar arguments should apply to other remote sensing applications as well.

There are many possible ways to implement aperture synthesis; and each configuration must be evaluated for its sensitivity. General formulas have been given in this paper which can apply to a wide variety of situations and antenna configurations.

\section{ACKNOWLEDGMENT}

The author wishes to acknowledge the contributions of J. Carr (ORI) and C. Ruf, A. Tanner and C. Swift (University of Massachusetts) and others with whom I have discussed these and related issues during the many years that this paper has been evolving. 


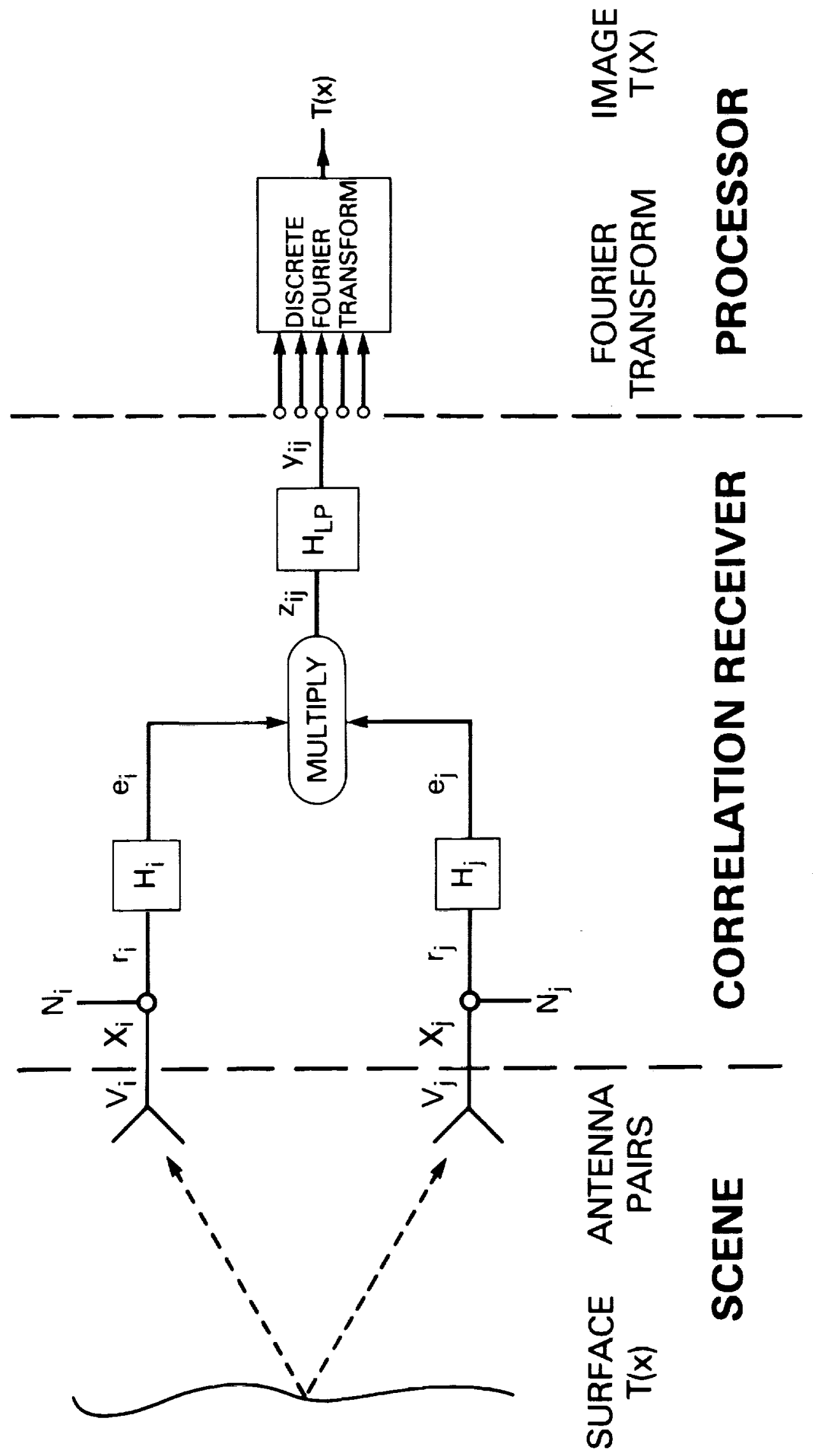




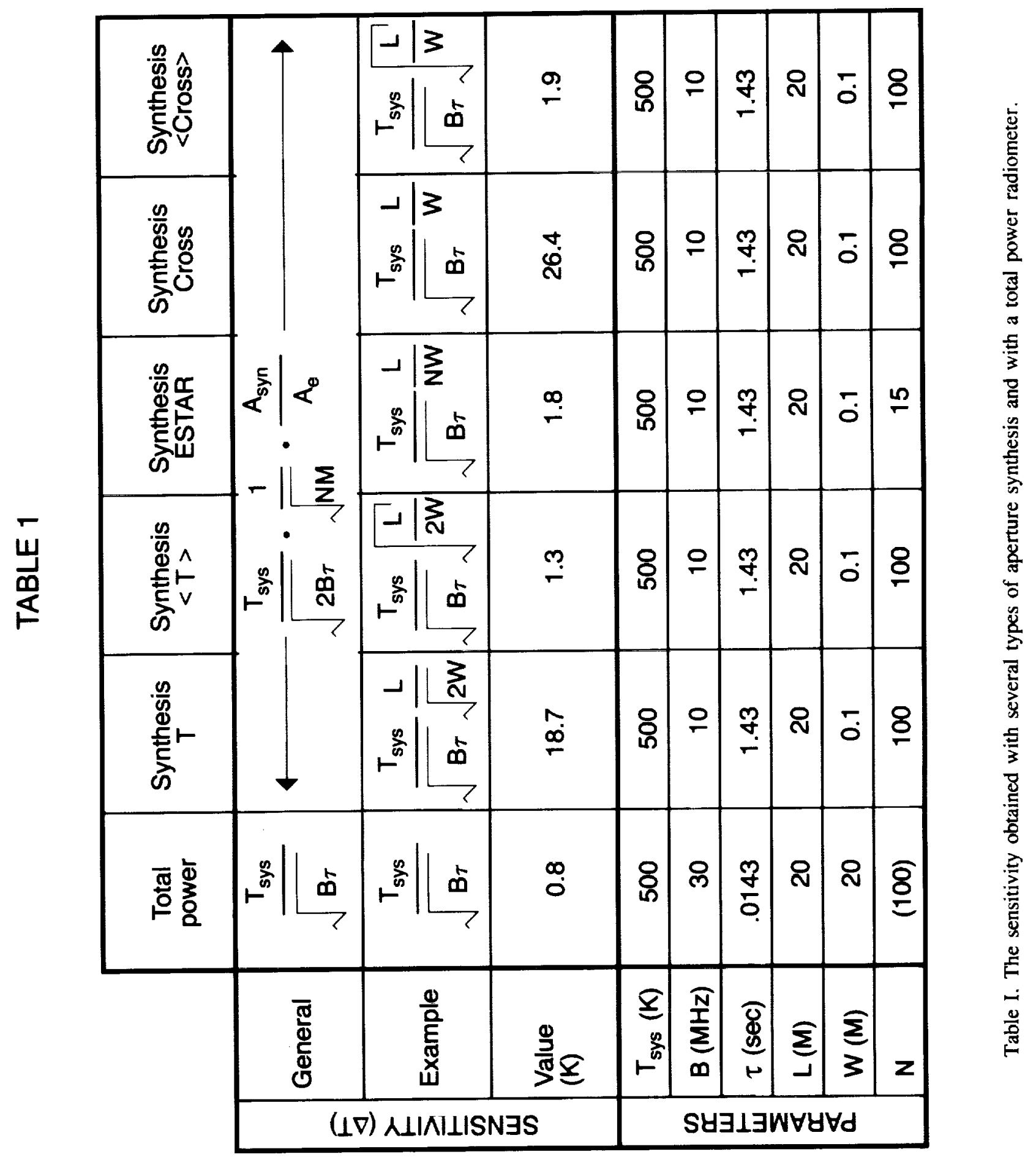




\section{REFERENCES}

Brouw, W.N. (1975), "Aperture Synthesis," in Methods in Computational Physics, Vol. 14, B. Adler, Ed., pp. 131-175, Academic Press.

Butler, D.M. et al. (1988), "From Pattern to Process: The Strategy of the Earth Observing System," Eos Science Steering Committee Report, Vol. II.

Collin, R.E. and F.J. Zucker (1969), Antenna Theory, Vol. 1, Chapter 4, McGraw-Hill, Inc.

Folamont, E.B. and M.C.H. Wright (1974), "Interferometry and Aperture Synthesis," in Galactic and Extragalactic Radio Astronomy, pp. 256-290, G.L. Verschuur and K.I. Kellerman, Ed., Springer-Verlag, New York.

Hewish, A. (1965), "The Synthesis of Giant Radio Telescopes," in Science Progress, Vol. 53, pp. 355-368.

Kraus, J.D. (1966), Radio Astronomy, McGraw-Hill Book, Inc.

Jordan, E.C. and K.G. Balmain (1968), Electromagnetic Waves and Radiating Systems, Chapter 12, Prentice-Hall, Englewood Cliffs, New Jersey.

Le Vine, D.M. and J. Good (1983), "Aperture Synthesis for Microwave Radiometers in Space," NASA TM-85033 (Avail. NTIS \#83N-36539).

Le Vine, D.M., T.T. Wilheit, R. Murphy and C. Swift (1989), "A Multifrequency Microwave Radiometer of the Future," IEEE Trans. on Geosci. \& Remote Sensing, Vol. 27 (2), pp. 193-199.

Mel'nik, Yu. A. (1972), "Space-Time Handling of Radiothermal Signals from Radiators that Move in the Near Zone of an Interferometer," Izvestiya Vysshikh Uchebnykh Zavedenii, Radiofisika., Vol. 15 (\#15), pp. 1376-1380.

Milman, A.S. (1988), “Sparse-Aperture Microwave Radiometers for Earth Remote Sensing, " Radio Science, Vol, 23 (\$2), pp. 193-205.

Moffet, A.T. (1968), "Minimum Redundancy Linear Arrays," IEEE Trans. on Antennas and Propagation, Vol. $16(\$ 2)$, pp. $172-175$.

Murphy, R., et al. (1987), "Earth Observing System Reports," Vol. Ile, report of the "High-Resolution Multifrequency Microwave Radiometer Instrument (HMMR) panel, NASA technical report, Washington, D.C.

Napier, P.J., A.R. Thompson and R.D. Ekers (1983), "The Very Large Array: Design and Performance of a Modern Synthesis Radio Telescope," Proceedings IEEE, Vol. 71 (11), pp. 1295-1320.

Ruf, C.S., C.T. Swift, A.B. Tanner and D.M. Le Vine (1988), "Interferometric Synthetic Aperture Microwave Radiometry for the Remote Sensing of the Earth," IEEE Trans. Geosci \& Remote Sensing, Vol. 26 (5), pp. 597-611.

Schanda, E. (1976), "High Ground Resolution in Passive Microwave Earth Observation from Space by Multiple Wavelength Aperture Synthesis," Congress, Internat. Astronautical Federation, Anaheim, California, October.

Schanda, E. (1979), "Multiple Wavelength Aperture Synthesis for Passive Sensing of the Earth's Surface," Symposium Digest, IEEE Antennas and Propagation Society Symposium, Seattle, Washington, pp. 762-763.

Swenson, G.W. and N.C. Mathur (1968), "The Interferometer in Radio Astronomy," Proceedings IEEE, Vol. 56 (12), pp. $2114-2130$.

Swift, C.T., L.S. Fedor and R.O. Ramseier (1985), "An Algorithm to Measure Sea Ice Concentration with Microwave Radiometers," J. Geophys. Res., Vol. 90 (Cl), pp. 1087-1099. 
Swift, C.T., C. Ruf, A. Tanner and D. Le Vine (1986), "The Electronically Steered Thinned Array Radiometer ESTAR," Proceedings, IGARSS '86 Symposium, Zurich, Switzerland, pp. 591-593 (Ref: ESA SP- 254, European Space Agency, Neuilly, France).

Thompson, A.R. and L.R. D’Addario (1982), "Frequency Response of a Synthesis Array: Performance Limitations and Design Tolerances," Radio Science, Vol. 17 (2), pp. 257-370.

Thompson, A.R., J.M. Moran and G.W. Swenson (1986), Interferometry and Synthesis in Radio Astronomy, J. Wiley \& Sons, New York.

Thomann, G.C. (1976), "Experimental Results of the Remote Sensing of Surface Salinity at 21-cm Wavelength," IEEE Trans. Geosci. Electronics, Vol. GE-14, pp. 198-214.

Tiuri, M.E. (1964), “Radio Astronomy Receivers,” IEEE Trans. Antennas and Propagation, Vol. AP-12, pp. 930-938.

Ulaby, F.T., R.K. Moore and A.K. Fung (1981), Microwave Remote Sensing, Addison-Wesley Publishing Company, Reading, Massachusetts.

Wang, J.R., P.E. O'Neill, T.J. Jackson and E.T. Engman (1983), "Multifrequency Measurements of the Effects of Soil Moisture, Soil Texture, and Surface Roughness," IEEE Trans. Geosci. and Remote Sensing, Vol. 21 (1), pp. 44-51.

Wilheit, T.T. and A.T.C. Chang (1980), "An Algorithm for Retrieval of Ocean Surface and Atmospheric Parameters from the Observations of the Scanning Multichannel Microwave Radiometer," Radio Science, Vol. 15, pp. 525-544. 


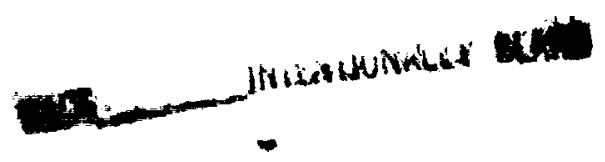




\section{APPENDIX A \\ THE MEAN AND VARIANCE OF THE OUTPUT OF A \\ CORRELATION RECEIVER}

In this appendix the mean and variance of the output $y(r)$ of the correlation receiver shown in Figure 1 will be computed. In doing so, the following notation will be used:

$$
S_{i j}(v)=\int_{-\infty}^{\infty} R_{i j}(t) \exp (j 2 \pi v t) d t
$$

where $R_{i j}(\tau)=\left\langle r_{i}(t) r_{j}(t+\tau)\right\rangle$ is the correlation function of the input voltages, $r_{i}(t)$, and the $S_{i j}(v)$ are the power spectra associated with these correlation functions. It will be assumed in all that follows that the $r_{i}(t)$ are stationary random processes. In describing the filters, lowercase symbols [e.g., h(t)] will be used to denote functions of time, and capital letters [e.g., $H(v)]$ will denote their Fourier transform. The symbol “ $*$ ” will be used to denote a convolution, except when used as a superscript when it will denote the complex conjugate.

\section{A. MEAN}

Referring to Figure 1 and letting $\left\langle y_{i j}(r)\right\rangle$ be the mean output of the radiometer when the input is $r$, one obtains:

$$
\begin{aligned}
<y_{i j}(r)> & =<e_{i} e_{j}>* h_{L P} \\
& =<\left[r_{i} * h_{i}\right]\left[r_{j} * h_{j}\right]>* h_{L P} \\
= & \int_{-\infty}^{\infty} R_{i j}\left(t^{\prime}-t^{\prime \prime}\right) h_{i}\left(t^{\prime \prime \prime}-t^{\prime}\right) h_{j}\left(t^{\prime \prime \prime}-t^{\prime \prime}\right) h_{L P}\left(t-t^{\prime \prime \prime}\right) d t^{\prime} d t^{\prime \prime} d t^{\prime \prime}
\end{aligned}
$$

Now, replacing all the terms in the integrand by their Fourier transform, one obtains:

$$
\left\langle y_{i j}(r)\right\rangle=H_{L P}(0) \int_{\infty}^{\infty} S_{i j}(v) H_{i}(v) H_{j}^{*}(v) d v
$$




\section{B. Variance}

The variance of the receiver output is: $\operatorname{Var}[y]=\left\langle y^{2}\right\rangle-\langle y\rangle$. Since the mean value, $\langle y\rangle$, is given above (Equation A3), it is necessary only to compute $\left\langle\mathrm{y}^{2}>\right.$ :

$$
\begin{aligned}
\left\langle y_{i j}^{2}(r)\right\rangle= & \left.<\left[z_{i j} * h_{L P}\right]\left[z_{i j} * h_{L P}\right]\right\rangle \\
& =\int_{-\infty}^{\infty} S_{z z}(v) H_{L P}{ }^{2}(v) d v
\end{aligned}
$$

where $S_{z z}(v)$ is the Fourier transform of the correlation function $R_{z z}(\tau)=\left\langle z_{i j}(t) z_{i j}(t+\tau)\right\rangle$; and the second line is obtained by substituting for the filter in terms of its Fourier transform as done above (e.g., Equations A2 and A3). Substituting for $z_{i j}(t)$ in terms of the signals in each arm of the receiver (i.e., $z_{i j}(t)=e_{i}(t) e_{j}(t)$; Figure 1), one obtains:

$$
\begin{aligned}
\mathrm{R}_{z z}(\tau)= & <\left[\mathrm{e}_{\mathrm{i}}(\mathrm{t}) \mathrm{e}_{\mathrm{j}}(\mathrm{t})\right]\left[\mathrm{e}_{\mathrm{i}}(\mathrm{t}+\tau) \mathrm{e}_{\mathrm{j}}(\mathrm{t}+\tau)\right]> \\
= & <\mathrm{e}_{\mathrm{i}}(\mathrm{t}) \mathrm{e}_{\mathrm{i}}(\mathrm{t}+\tau)><\mathrm{e}_{\mathrm{j}}(\mathrm{t}) \mathrm{e}_{\mathrm{j}}(\mathrm{t}+\tau)> \\
& +<\mathrm{e}_{\mathrm{i}}(\mathrm{t}) \mathrm{e}_{\mathrm{j}}(\mathrm{t})><\mathrm{e}_{\mathrm{i}}(\mathrm{t}+\tau) \mathrm{e}_{\mathrm{j}}(\mathrm{t}+\tau)> \\
& +<\mathrm{e}_{\mathrm{i}}(\mathrm{t}+\tau) \mathrm{e}_{j}(\mathrm{t})><\mathrm{e}_{\mathrm{i}}(\mathrm{t}) \mathrm{e}_{\mathrm{j}}(\mathrm{t}+\tau)>
\end{aligned}
$$

where the last step is obtained by assuming that the $e_{i}(t)$ are Gaussian random processes. Now, following the same procedure as used above to calculate the mean, one obtains the following result for each of the pairs above:

$$
\begin{aligned}
\left\langle e_{i}(t) e_{j}(t+\tau)\right\rangle= & \left\langle\left[r_{i}(t) * h_{i}(t)\right]\left[r_{j}(t+\tau) * h_{j}(t+\tau)\right]\right\rangle \\
& =\int_{-\infty}^{\infty} S_{i j}(v) H_{i}(v) H_{j}^{*}(v) \exp (-j 2 \pi v \tau) d v
\end{aligned}
$$

Equation A6 applies to any combination (i,j): that is, for $\left\langle e_{i} e_{j}\right\rangle$ as well as $\left\langle e_{i} e_{i}\right\rangle$ and $\left\langle e_{j} e_{j}\right\rangle$, etc. Now, taking the Fourier transform of Equation A5 using the results of Equation A6 above, one obtains:

$$
\begin{aligned}
S_{z z}(v) & =\delta(v-0)\left\{\int_{-\infty}^{\infty} S_{i j}(v) H_{i}(v) H_{j}^{*}(v) d v\right\}^{2} \\
& +\left[S_{i j}(v) H_{i}(v) H_{i}^{*}(v)\right] *\left[S_{i j}(v) H_{j}(v) H_{j}^{*}(v)\right] \\
& +\left[S_{i j}(v) H_{i}(v) H_{j}^{*}(v)\right] *\left[S_{i j}(v) H_{i}(v) H_{j}^{*}(v)\right] \\
& =S^{a}{ }_{z z}(v)+S^{b}{ }_{z z}(v)+S^{c}{ }_{z z}(v)
\end{aligned}
$$


Substituting this result (Equation A7) into Equation A4 and comparing with Equation A3, one obtains:

$$
\left\langle\mathrm{y}_{\mathrm{ij}}{ }^{2}(\mathrm{r})\right\rangle=\left\langle\mathrm{y}_{\mathrm{ij}}(\mathrm{r})\right\rangle^{2}+\int_{-\infty}^{\infty}\left[\mathrm{S}_{z \mathrm{z}}^{\mathrm{a}}(\mathrm{v})+\mathrm{S}_{\mathrm{zz}}^{\mathrm{b}}(\mathrm{v})\right] \mathrm{H}_{\mathrm{LP}}{ }_{\mathrm{LP}}(\mathrm{v}) \mathrm{dv}
$$

It follows from Equation $\mathrm{A} 8$ that:

$$
\begin{aligned}
\operatorname{Var}\left[y_{i j}(r)\right] & =\int_{-\infty}^{\infty}\left[S^{b}{ }_{z z}(v)+S^{c}{ }_{z z}(v)\right] H^{2}{ }_{L P}(v) d v \\
& =\left[S^{b}{ }_{z z}(0)+S^{c}{ }_{z z}(0)\right] \int_{-\infty}^{\infty} H^{2}(v) d v
\end{aligned}
$$

The last step in Equation A9 follows because it has been assumed that $\mathrm{H}_{\mathrm{LP}}(v)$ only passes signals in a narrow band very close to $v=0$. Finally, substituting for the $S_{z z}(0)$ from Equation $A 7$, one obtains:

$$
\operatorname{Var}\left[y_{i j}(r)\right]=\int_{-\infty}^{\infty}\left[S^{2}{ }_{i j}(v)+S_{i j}(v) S_{j j}(v)\right] H^{2}{ }_{i}(v) H^{2}{ }_{j}(v) d v \int_{-\infty}^{\infty} H^{2}{ }_{L P}(v) d v
$$

The mean and variance of the output of the correlation receiver shown in Figure 1 are given in Equations A3 and A 10 for a reasonably general set of assumptions. They simplify somewhat in the important, special case when the system transfer functions $H_{i}(v)$ are very narrow compared to the spectra of the input signal, $S_{i j}(v)$, which is the situation encountered in systems designed for microwave remote sensing of the earth from space. In this narrow bandwidth case, the signal spectra can be factored out of the integrals and one obtains:

$$
\begin{aligned}
& <y_{i j}(r)>=S_{i j}\left(v_{v}\right) H_{L P}(0) \int_{-\infty}^{\infty} H_{i}(v) H_{j}^{*}(v) d v \\
& \operatorname{Var}\left[y_{i j}(r)\right]=\left[S^{2}{ }_{i j}\left(v_{0}\right)+S_{i i}\left(v_{0}\right) S_{i j}\left(v_{i j}\right)\right] \cdot \int_{-\infty}^{\infty} H^{2}{ }_{i}(v) H^{2}{ }_{j}(v) d v \int_{-\infty}^{\infty} H^{2}{ }_{L P}(v) d v
\end{aligned}
$$

where $v_{0}$ is the center frequency of the filters, $H_{i}(v)$. Equations $A 11$ and $A 12$ are the ones used in the text to compute the signal-to-noise ratio of the receiver output. 


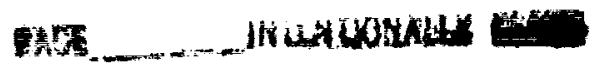




\section{APPENDIX B}

\section{POWER SPECTRUM OF THE ANTENNA OUTPUT VOLTAGES}

It is the objective of this appendix to derive an expression for the spectrum of the antenna output voltages (Figure 1) which are the inputs to the arms of the correlation receiver. In the notation employed in the text this spectrum has been denoted, $\left\langle<\mathrm{X}_{\mathrm{i}} \mathrm{X}_{\mathrm{j}}\right\rangle>$, where the double brackets $\left\langle<>>\right.$ denote a Fourier transform and $\mathrm{X}_{\mathrm{i}} \mathrm{X}_{\mathrm{j}}$ is a shorthand notation for the correlation function $\left\langle\mathrm{X}_{\mathrm{i}}(\mathrm{t}) \mathrm{X}_{\mathrm{j}}(\mathrm{t}+\tau)>\right.$. (In the text, a distinction was made between the response, $\mathrm{X}^{\circ}$, to a uniform scene and the response, $x_{i}$, to fluctuations about this background. In this case one has: $X_{i}=X^{\circ}+x_{i}$. This distinction is not being made in this appendix and $X_{i}$ is the response of the antenna to an arbitrary scene.) Referring to Figure 1 , the $X_{i, j}$ are the same as the voltages, $\mathrm{V}_{\mathrm{i}, \mathrm{j}}$, at the antenna output terminals; and the discussion in this appendix will be in terms of the voltages, $V\left(r_{i}, t\right)$.

In this appendix, let $V\left(r_{i}, t\right)$ denote the time-dependent voltage at the output terminal of the antenna at position $r_{i}$; and using the same notation as adopted in Appendix A; let $S_{\mathrm{ij}}(\mathrm{v})$ ) be the Fourier transform of the correlation function $\mathrm{R}_{\mathrm{ij}}(\tau)=$ $\left\langle V\left(r_{i},(t+\tau)\right) V^{*}\left(r_{j, l}\right)\right\rangle$. In the special case when the voltages are stationary random processes, one has $S_{i j}(v)=$ $\left\langle V\left(r_{i}, v\right) V^{*}\left(r_{j}, v\right)\right\rangle$ where the $V\left(r_{i}, v\right)$ are the Fourier-Stieltjes transforms of the antenna voltages.

In order to obtain expressions for the $\mathrm{V}\left(\mathrm{r}_{\mathrm{i}}, \mathrm{v}\right)$, imagine two parallel planes, the scene plane which is the source of the radiation (e.g., the surface of the earth) and a second plane called the image plane at a height $H_{o}$ above the scene plane. The image plane contains all the interferometer antenna pairs. Let the coordinate origin be in the scene plane and let $\underline{r}^{\prime}=+x^{\prime} \hat{x}+y^{\prime} \hat{y}+z^{\prime} \hat{z}$ denote a position in the scene plane $\left(z^{\prime}=0\right)$ and $\underline{r}_{i}=x_{i} \hat{x}+y_{i} \hat{y}+z_{i} \hat{z}$ denote the position of an antenna in the image plane $\left(\mathrm{z}_{\mathrm{i}}=\mathrm{H}_{\mathrm{o}}\right)$. The geometry is illustrated in Figure B1.

It is desired to obtain an expression for the output voltage $V\left(\underline{r}_{i}, v\right)$ from the antenna at $\underline{r}_{i}$ in terms of the electric field, $\underline{E}_{s}\left(r_{i}^{\prime}, v\right)$, on the scene plane. To begin, consider radiation from a small patch $\Delta x^{\prime} \Delta y^{\prime}$ at $I^{\prime}$ in the scene plane. The electric field $\Delta \underline{E}\left(\underline{r}_{i} / \underline{r}^{\prime}, v\right)$ radiated from this small patch to the antenna at $\underline{\underline{r}}_{i}$ can be obtained from the vector Helmholtz equation (by assuming that the patch is a small aperture in an otherwise opaque screen on which the tangential components of the fields are zero). Following standard procedures (e.g., Tai, 1971), one obtains:

$$
\Delta \underline{E}\left(\underline{r}_{i} / \underline{r}^{\prime}, v\right)=-\left\{\left[\hat{n} \times \bar{\nabla} \times \underline{E}_{s}\left(\underline{r}^{\prime}, v\right)\right] \cdot \overline{\bar{G}}\left(\underline{r}^{\prime} / \underline{r}_{i}\right)+\left[\hat{n} \times \underline{E}_{s}\left(\underline{r}^{\prime}, v\right)\right] \cdot \bar{\nabla} \times \overline{\bar{G}}\left(\underline{r}^{\prime} / \underline{r}_{i}\right)\right\} \Delta x^{\prime} \Delta y^{\prime}
$$

where $\hat{n}=\hat{z}$ is the unit vector normal to the scene plane and $\overline{\bar{G}}\left(\underline{r}_{i} / \underline{r}^{\prime}\right)$ is a dyadic Green's function satisfying the free space wave equation in the region $z^{\prime}>0$. A convenient choice for $G\left(\underline{r}_{i} / \mathbf{r}^{\prime}\right)$ is (Tai, 1971; Section 18): 


$$
\overline{\bar{G}}\left(\underline{r}_{i} / \underline{\underline{r}}\right)=\left[\overline{\bar{I}}-1 / k^{2} \nabla \nabla\right]\left[g_{0}\left(r_{i} / \underline{r}\right)-g_{0}\left(\underline{r}_{i} / \underline{\underline{r}}\right)\right]-2 \hat{z} \hat{z} g_{0}\left(r_{i} / \tilde{r}^{\prime}\right)
$$

where $\overline{\overline{\mathrm{I}}}$ is the unit dyadic, $\mathrm{g}_{0}\left(\underline{\underline{r}} / \underline{\underline{r}}^{\prime}\right)=\exp \left(\mathrm{jk}\left|\underline{r}-\underline{r}^{\prime}\right|\right) / 4 \pi\left|\underline{r}-\underline{r}^{\prime}\right|$ is the free space scalar Green's function and $\tilde{\underline{\mathbf{r}}}=\underline{\mathbf{r}}-$ $2 z \hat{z}$ is the "image" of a point at $\underline{\underline{r}}$ behind the scene plane. The following properties apply to this Green's function when the source point $\underline{r}^{\prime}$ is on the scene plane:

$$
\begin{aligned}
& \hat{\mathbf{n}} \times \overline{\bar{G}}\left(\mathbf{r}^{1 / \underline{r})}=0\right. \\
& \bar{\nabla}^{\prime} \times \overline{\bar{G}}\left(\underline{r}^{\prime} / \underline{r}\right)=\bar{\nabla}^{\prime} \times \overline{\bar{I}} g_{0}\left(\underline{r}^{\prime} / \underline{r}\right)-\bar{\nabla}^{\prime} \times \overline{\bar{I}}_{i} g_{0}\left(\tilde{r}^{\prime} / \mathbf{r}\right) \\
& (\hat{\mathrm{n}} \times \underline{\mathrm{E}}) \cdot \bar{\nabla}^{\prime} \times G\left(\underline{r}^{\prime} / \underline{r}\right)=2(j \mathrm{k}-1 / R) g_{0}\left(\underline{r} / \underline{r}^{\prime}\right)[\nabla R \times(\underline{E} \times \hat{z})] \\
& \overline{\overline{I_{i}}}=\overline{\overline{\mathrm{I}}}-2 \hat{\mathbf{z}} \hat{\mathrm{z}}
\end{aligned}
$$

Now, noting that $[\hat{\mathrm{n}} \times(\overline{\bar{\nabla}} \times \underline{\mathrm{E}})] \cdot \overline{\overline{\mathrm{G}}}=-(\overline{\bar{\nabla}} \times \underline{\mathrm{E}}) \cdot[\hat{\mathrm{n}} \times \overline{\overline{\mathrm{G}}}]$ and using the preceeding properties of the dyadic Green's function, one may write:

$$
\Delta E\left(\underline{r}_{i} / \underline{r}^{\prime}, v\right)=2(j k-1 / R) g_{0}\left(\underline{r}_{i} / \underline{r}^{\prime}\right)\left[\nabla R \times\left(\underline{E}_{s} \times \hat{z}\right)\right]
$$

The expression in Equation B4. is the signal radiated from the patch on the scene plane to an antenna at $\underline{r}_{i}$. The response of an antenna to radiation from the small patch can be expressed in terms of the voltage transfer function of the antenna, $\underline{A}\left(\underline{r} / \underline{r}^{\prime}, v\right)$, as follows:

$$
\Delta \mathrm{V}\left(\underline{\mathrm{r}} / \underline{\mathbf{r}}^{\prime}, \mathrm{v}\right)=\Delta \underline{\mathrm{E}}\left(\underline{\mathrm{r}} / \underline{\mathbf{r}}^{\prime}, \mathrm{v}\right) \cdot \underline{\mathrm{A}}\left(\underline{\mathrm{r}} / \underline{\underline{r}}^{\prime}, \mathrm{v}\right)
$$

where the voltage transfer function is a vector quantity to account for the polarization of the antenna (Collin and Zucker, 1969) and can be obtained from the reciprocity theorem for antennas. One can show that $\underline{A}\left(r / \underline{r}^{\prime}, v\right) \cdot \underline{A} \underline{A}^{*}\left(\underline{r} / \underline{r}^{\prime}, v\right)=$ $A_{e} P_{n}(\underline{r} / \underline{r})$ where $A_{e}$ is the effective area of the receiving antenna and $P_{n}(r / \underline{r})$ is the normalized power pattern of the antenna (Kraus, 1966).

To obtain the response of the antenna to all sources, it is now necessary to sum over all of the scene plane. In the limit of infinitesimally small patches, the sum approaches an integral and one obtains:

$$
\begin{aligned}
& V\left(\underline{r}_{i}, v\right)=\int \Delta \underline{E}\left(\underline{r}_{i} / \underline{r}^{\prime}, v\right) \cdot \underline{A}\left(\underline{r}_{i} / \underline{r}^{\prime}, v\right) d \underline{r}^{\prime} \\
& =2 \int \underline{E}_{0}\left(\underline{r}^{\prime}, v\right) \cdot \underline{A}\left(\underline{r}_{i} / \underline{r}^{\prime}\right)\left[j k-1 / R_{i}\right]\left[z_{i} / R_{i}\right] g_{0}\left(\underline{r}_{i} / \underline{r}^{\prime}\right) d \underline{r}^{\prime}
\end{aligned}
$$

where the radiated electric field in Equation B5c has been expressed in terms of it components, $\underline{E}_{o}\left(\underline{I}^{\prime}, v\right)=e_{h} \hat{h}+e_{v} \hat{v}$, along horizontally and vertically polarized unit vectors $(\hat{\mathrm{h}}, \hat{\mathrm{v}})$. These unit vectors are defined locally (i.e. at each point $\underline{\mathbf{r}}$ ) 
with respect to the plane formed by the normal to the surface $(\hat{\mathbf{z}})$ and the vector, $\underline{\mathbf{R}}_{i}=\underline{\mathbf{r}}_{i}-\underline{\mathbf{r}}^{\prime}$, from the antenna at $\underline{\underline{r}}_{\mathrm{i}}$ to the source point at $\underline{\mathbf{r}}^{\prime}$. The unit vectors are:

$$
\begin{aligned}
& \hat{\mathrm{h}}=\left(\hat{z} \times \nabla \mathrm{R}_{\mathrm{i}}\right) / \sin \left(\theta_{\mathrm{i}}\right) \\
& \hat{\mathrm{v}}=\nabla \mathrm{R}_{\mathrm{i}} \times\left(\hat{\mathrm{z}} \times \nabla \mathrm{R}_{\mathrm{i}}\right) / \sin \left(\theta_{\mathrm{i}}\right)
\end{aligned}
$$

where $\sin \left(\theta_{i}\right)=\left|\hat{z} \times \nabla R_{i}\right|$ is the angle between the line-of-sight and the $z$-axis. The projections of the electric field onto these unit vectors $\left(e_{h}, e_{v}\right)$ can be expressed in terms of the field on the surface, $\underline{E}_{s}\left(\underline{r}^{\prime}, v\right)$, as follows:

$$
\begin{aligned}
\mathrm{e}_{\mathrm{h}} & =\underline{\mathrm{E}}_{\mathrm{s}} \cdot \hat{\mathrm{h}} \cos \left(\theta_{\mathrm{i}}\right) \\
\mathrm{e}_{\mathrm{v}} & =\left[\left(\underline{\mathrm{E}}_{\mathrm{s}} \cdot \hat{\mathrm{v}}\right)-\left(\underline{\mathrm{E}}_{\mathrm{s}} \cdot \nabla \mathrm{R}_{\mathrm{i}}\right) \tan \left(\theta_{\mathrm{i}}\right)\right] \cos \left(\theta_{\mathrm{i}}\right)
\end{aligned}
$$

In Equation B4 the factor, $\cos \left(\theta_{i}\right)$, appearing above has been factored out and appears explicitly in the form $\cos \left(\theta_{i}\right)=z_{i} / R_{i}$ where $R_{i}$ is the magnitude of the vector $\underline{R}_{i}$ and $z_{i}=H_{o}$ (Figure B1). Hence, in Equation B5b one has $e_{h}=\underline{E}_{s} \cdot \hat{h}$ and $e_{v}$ $=\underline{E}_{s} \cdot \hat{v}-\left(E_{s} \cdot \nabla R_{1}\right) \tan \left(\theta_{1}\right)$.

Now, using these results and assuming that the distance between the image plane and scene plane is much larger than the wavelength of any radiation in the passband of interest (i.e., $k R_{i}>>1$ for all $k$ of interest) and that the fields on the surface are (spatially) uncorrelated, one obtains:

$$
S_{i j}(v)=\left\langle V\left(\underline{r}_{i}, v\right) V^{*}\left(\underline{r}_{j}, v\right)\right\rangle=\left(k_{v} / 2 \pi\right)^{2} \int\left(H_{o} / R_{i} R_{j}\right)^{2}\left\langle\underline{A}\left(\underline{r}_{i} / \underline{r}^{\prime} \cdot v\right) \cdot \overline{\bar{I}}\left(\underline{r}^{\prime} \cdot v\right) \cdot A^{*}\left(\underline{r}_{j} / \underline{r}, v\right)\right\rangle_{p} \exp \left(j k\left[R_{i}-R_{j}\right]\right) d \underline{r}^{\prime}
$$

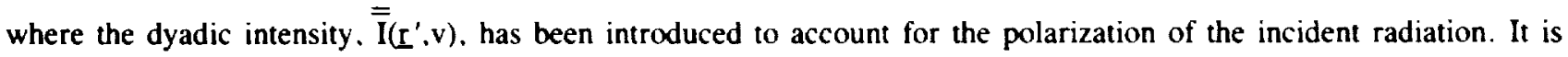
defined as follows:

$$
\overline{\bar{I}\left(r^{\prime} . v\right)}=\left[\begin{array}{ll}
I_{h h} \hat{h} \hat{h} & I_{h v} \hat{v} \hat{h} \\
I_{h v} \hat{h} \hat{v} & I_{w}, \hat{v} \hat{v}
\end{array}\right]
$$

where $I_{a b}=\left\langle e_{a}\left(r^{\prime}, v\right) e_{b}^{*}\left(r^{\prime}, v\right)\right\rangle$. The brackets $\left\langle>\right.$ in the definition for $I_{a b}$ denote a statistical average over the spatial variable of the random process, and the brackets $<>_{p}$ in Equation B7 indicate an average over polarization states (Collin and Zucker, 1969).

In the practical cases which are of interest in remote sensing from space, the distance between the scene and image planes is much larger than the distance between the receiving antennas. In this case one may approximate $R_{\mathbf{l}} R_{\mathbf{l}}$ in the 
denominator in Equation $B 7$ by $R_{i} R_{j}=R_{o}{ }^{2}$, and for the factor $R_{i}-R_{j}$ appearing in the exponential, one may write (Appendix C):

$$
R_{i}-R_{j}=\left|\underline{r}_{i}-\underline{r}^{\prime}\right|-\left|\underline{r}_{j}-\underline{r}_{i}\right| \cong\left(x_{i}-x_{j}\right) \cos \left(\alpha_{x}\right)+\left(y_{i}-y_{j}\right) \cos \left(\alpha_{y}\right)
$$

where $\cos \left(\alpha_{x, y}\right)$ are the direction cosines of the vector from the source point at $\underline{r}^{\prime}$ in the scene plane to the origin in the image plane. Using this approximation, one obtains:

$$
S_{i j}(v)=\int\left[k_{0} H_{o} / 2 R_{o}{ }^{2}\right]^{2}<\underline{A}\left(\underline{r}_{i} / \underline{r}^{\prime}, v\right) \cdot \overline{\bar{I}}\left(\underline{r}^{\prime}, v\right) \cdot \underline{A}^{*}\left(\underline{r}_{j} / \underline{r}^{\prime}, v\right)>_{p} \exp \left[j 2 \pi\left(\eta_{x} \cos \alpha_{x}+\eta_{y} \cos \alpha_{y}\right)\right] d \underline{r}^{\prime}
$$

where

$$
\begin{aligned}
& \eta_{x}=\frac{x_{i}-x_{j}}{c} v \\
& \eta_{y}=\frac{y_{i}-y_{j}}{c} v \\
& R_{o}=\left|H_{o} \hat{z}-\underline{r}^{\prime}\right|
\end{aligned}
$$

It is convenient to do the integration in Equation $\mathrm{B} 10$ in terms of the direction $\operatorname{cosines} \cos \left(\alpha_{\mathrm{x}, \mathrm{y}}\right)$ rather than in terms of the coordinates $\underline{r}^{\prime}$. Thus, making the following change of variables:

$$
\begin{aligned}
& q_{x}=\cos \left(\alpha_{x}\right)=x^{\prime} / R_{0}\left(x^{\prime}, y^{\prime}\right) \\
& q_{y}=\cos \left(\alpha_{y}\right)=y^{\prime} / R_{0}\left(x^{\prime}, y^{\prime}\right)
\end{aligned}
$$

and using the Jacobian of this transformation $\mathrm{dq}_{\mathrm{x}} \mathrm{dq_{y }}=\left[\mathrm{H}_{\mathrm{o}} / \mathrm{R}_{\mathrm{v}}{ }^{2}\right]^{2} \mathrm{dx} \mathrm{x}^{\prime} \mathrm{dy} \mathrm{y}^{\prime}$, one obtains:

$$
\left.S_{i j}(v)=\left(1 / \lambda^{2}\right) \int_{-1}^{+1}<\underline{A} \cdot \overline{\bar{I}} \cdot \underline{A}^{*}\right\rangle_{p} \exp (j 2 \pi \underline{\eta} \cdot \underline{q}) d q
$$

where $\underline{\eta}=\eta_{x} \hat{x}+\eta_{y} \hat{y}$ and $q=q_{x} \hat{x}+q_{y} \hat{y}$.

A special case of importance is that in which the antennas are identical and the radiation is unpolarized. Ignoring the slight difference in location of the antennas (i.e., assuming that $\left.\underline{A}\left(r_{i} / \underline{r}^{\prime}, v\right)=\underline{A}\left(r_{j} / \underline{r}^{\prime}, v\right)\right)$, one obtains:

$$
\left.<\underline{A} \cdot \overline{\bar{I}} \cdot \underline{A}^{*}\right\rangle_{p}=(1 / 2) A_{e} P_{n}(\underline{q}) I_{p p}(\underline{q})
$$

where $P_{n}(\underline{q})$ is the normalized power pattern of the antenna in the direction $\underline{q}$ (Kraus, 1966), $A_{c}$ is its effective receiving 
area, and $I_{p p}(\underline{q})$ is the spectrum of the electric field on the scene plane with polarization $\hat{p}: I_{p p}(q)=\left\langle\left[\hat{p} \cdot \underline{E}_{o}(\underline{r}, v)\right]^{2}\right\rangle_{p}$ Hence, in this case:

$$
S_{i j}(v)=\left(A_{e} / 2 \lambda^{2}\right) \int_{-1}^{+1} P_{n}(\underline{q}) I_{p p}(\underline{q}) \exp (j 2 \pi \underline{\eta} \bullet \underline{q}) d \underline{q}
$$

In Appendix $E$ it is shown that $I_{p p}(q) /\left(Z_{0} \lambda^{2}\right)$ is the brightness in the direction $q$. Hence,

$$
S_{i j}(v) / Z_{o}=\left(A_{e} / 2\right) \int_{-1}^{+1} P_{n}(q) \tilde{B}(q) \exp (j 2 \pi \underline{\eta} \cdot q) d q
$$

where the definition $\widetilde{B}(q) d \underline{q}=B(\Omega) d \Omega$ has been made $(d \Omega=\sin (\theta) d \theta d \phi$ denotes the "solid angle" in spherical coordinates). It is also shown in Appendix $E$ that $\widetilde{B}(q)=B(q) / \sqrt{1-q_{x}{ }^{2}-q_{y}{ }^{2}}$. Hence, one may write:

$$
S_{i j}(v) / Z_{o}=\left(A_{e} / 2\right) \int_{-1}^{+1} \frac{P_{n}(q) B(q)}{\sqrt{1-q_{x}^{2}-q_{y}^{2}}} \exp (j 2 \pi \underline{\eta} \bullet q) d q
$$

where $\mathrm{B}(\underline{\mathrm{q}})$ is now the conventional definition of brightness and Equation $\mathrm{B} 17$ is the same as has appeared in the literature (e.g., Napier et al., 1983). Also, notice that $S_{i j}(v) / Z_{o}$ is the fraction of the power incident on the receiving antenna (per unit bandwidth) which is available at the antenna output terminals. It is the power which can be delivered to a load properly matched to the antenna (e.g., Collin and Zucker, 1969).

\section{REFERENCES:}

Collin, R.E. and F.J. Zucker (1969), Antenna Theory, Vol. I, Chapter 4, McGraw-Hill Inc.

Kraus, J.D. (1966), Radio Astronomy, Chapter 6, McGraw-Hill Inc.

Tai, C.T. (1971), Dyadic Green's Functions in Electromagnetic Theory, Intext, Pennsylvania. 


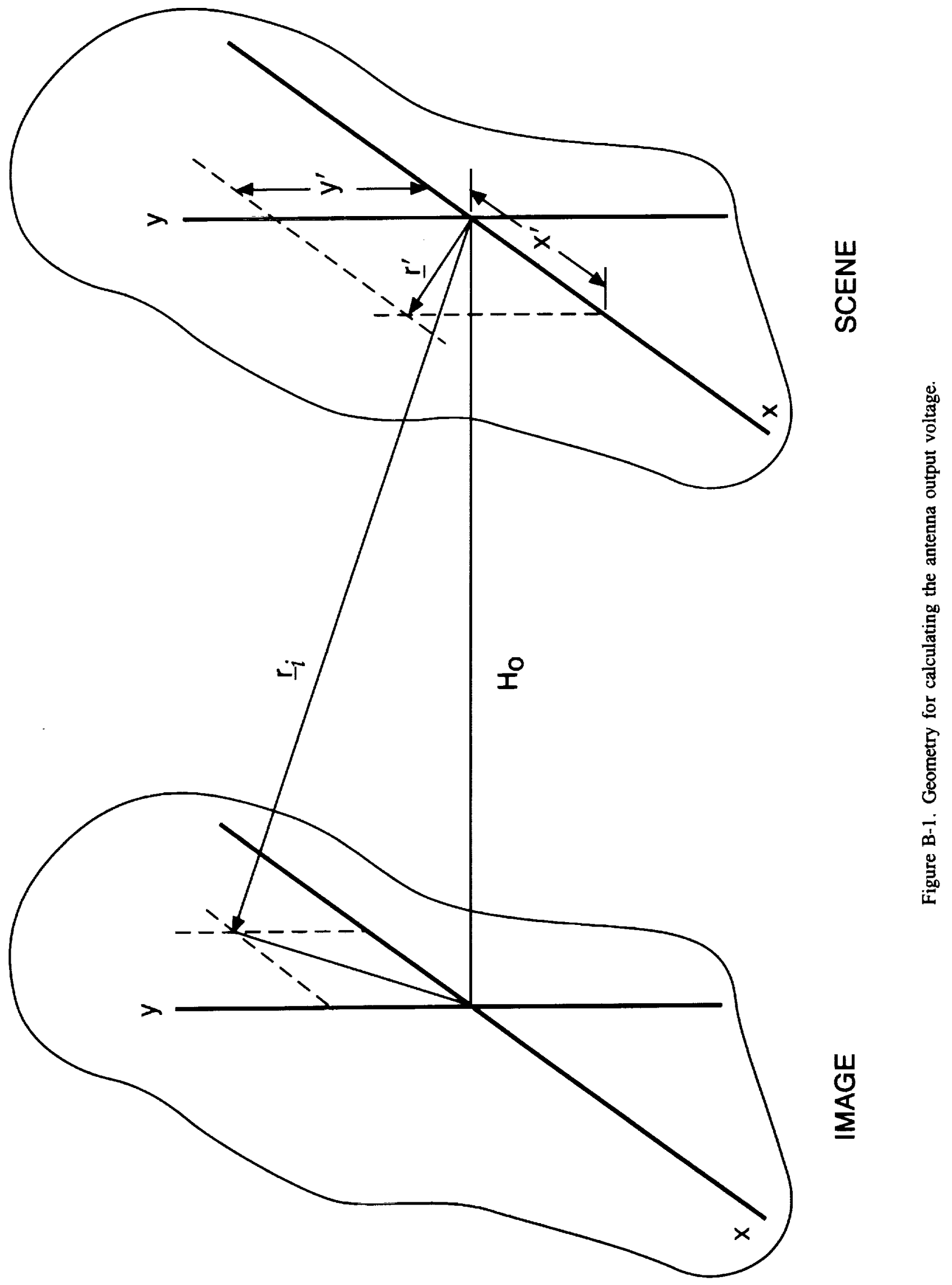




\section{APPENDIX C}

\section{THE SAGITTAL APPROXIMATION}

As defined in the previous discussion (Appendix B), $\underline{r}_{i}=x_{i} \hat{x}+y_{i} \hat{y}+H_{o} \hat{z}$ is the position vector of an antenna in the image plane, $\underline{r}^{\prime}=x^{\prime} \hat{x}+y^{\prime} \hat{y}$ is the position vector of an arbitrary point on the scene plane, and $\underline{R}\left(\underline{r}_{i} / \underline{r}^{\prime}\right)=\underline{r}_{i}-\underline{r}^{\prime}$ is the vector between the point $\underline{\mathbf{n}}^{\prime}$ on the scene plane and the antenna. In this appendix an approximate expression for $R\left(\underline{r}_{i} / \underline{r}\right)-R\left(\underline{r}_{j} / \underline{r}\right)$ will be derived where $R=|\underline{R}|$.

Letting $\underline{R}_{0}=H_{0} \hat{z}-\underline{r}^{\prime}$ and assuming that $\underline{r}_{t i} \cdot \underline{r}_{1 j}<R_{o}{ }^{2}$ where $\underline{r}_{t i}=x_{i j} \hat{x}+y_{i} \hat{y}$, one can write:

$$
\begin{aligned}
& R\left(\underline{r}_{i} / \underline{r}^{\prime}\right)-R\left(\underline{r}_{j} / \underline{r}^{\prime}\right)=\left|\underline{r}_{i}-\underline{r}^{\prime}\right|-\left|\underline{r}_{j}-\underline{\mathbf{r}}^{\prime}\right| \\
& =\left|\underline{\mathbf{R}}_{0}+\underline{\mathbf{r}}_{\mathrm{i}}\right|-\left|\underline{\mathbf{R}}_{\mathrm{o}}-\underline{\mathbf{r}}_{\mathrm{tj}}\right| \\
& =\sqrt{R_{o}^{2}+r_{t i}^{2}-2 \underline{R}_{0} \cdot \underline{I}_{t i}}-\sqrt{R_{o}^{2}+r_{t j}^{2}-2 \underline{R}_{0} \cdot \underline{r}_{t j}} \\
& =R_{\mathrm{o}} \sqrt{1+\left(\mathrm{r}_{\mathrm{ti}}{ }^{2} / \mathrm{R}_{\mathrm{o}}{ }^{2}\right)-2 \underline{\mathrm{R}}_{\mathrm{o}} \cdot \underline{\mathrm{r}}_{\mathrm{ti}} / \mathbf{R}_{\mathrm{o}}{ }^{2}}-\mathrm{R}_{\mathrm{o}} \sqrt{1+\left(\mathrm{r}_{\mathrm{ij}}{ }^{2} / \mathbf{R}_{\mathrm{o}}{ }^{2}\right)-2 \underline{\mathrm{R}}_{\mathrm{o}} \cdot \underline{-}_{\mathrm{ij}} / \mathbf{R}_{\mathrm{o}}{ }^{2}} \\
& \cong\left(r_{i i}^{2}-r_{i j}^{2}\right) / 2 R_{o}+\underline{R}_{0} \cdot\left(r_{t j}-\underline{r}_{t i}\right) / R_{o} \\
& \cong \underline{\mathbf{R}}_{\mathrm{o}} \cdot\left(\underline{\mathbf{r}}_{\mathrm{tj}}-\underline{\mathbf{r}}_{\mathrm{i}}\right) / \mathbf{R}_{\mathrm{o}} \\
& =\left(x_{i}-x_{j}\right) \cos \left(\alpha_{x}\right)+\left(y_{i}-y_{j}\right) \cos \left(\alpha_{y}\right)
\end{aligned}
$$

where $\cos \left(\alpha_{x, y}\right)$ are the direction cosines of $\underline{R}_{\mathrm{o}}$ (a vector from the source point to the origin in the image plane) with respect to the coordinate axes in the image plane $\left[\cos ^{2}\left(\alpha_{x}\right)+\cos ^{2}\left(\alpha_{y}\right)+\cos ^{2}\left(\alpha_{z}\right)=1\right]$.

In order to use this approximation in the exponential in Equation B7, the error made in replacing Equation C4 by Equation $\mathrm{C} 6$ must be small. Hence, one must have $k\left(r_{i}{ }^{2}-r_{j}^{2}\right) / R_{o}<<\pi$. As an upper limit on this error one can replace $\left(r_{i}^{2}-r_{j}^{2}\right)$ with $L^{2}$ where $L$ is the maximum spacing between antennas. Hence, the approximation requires that the size of the array in the image plane (squared) divided by the minimum distance to the scene plane be much less than a wavelength. This is a condition easily met in microwave remote sensing of the earth from space (e.g., even in an extreme case $\mathrm{L}$ is not likely to be more than a few 10 's of meters, but even in a very low orbit, $\mathrm{R}_{\mathrm{o}}$ is on the order of $300 \mathrm{~km}$ or more). 


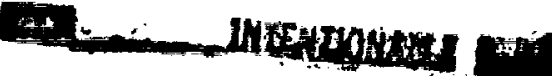




\section{APPENDIX D}

\section{COORDINATE TRANSFORMATIONS}

In this appendix the transformation between the Cartesian coordinates $\left(x^{\prime}, y^{\prime}, z^{\prime}\right)$ and the direction cosines $\left(q_{x}=\right.$ $\left.\cos \left(\alpha_{\mathrm{x}}\right) ; \mathrm{q}_{\mathrm{y}}=\cos \left(\alpha_{\mathrm{y}}\right) ; \mathrm{q}_{\mathrm{z}}=\cos \left(\alpha_{\mathrm{z}}\right)\right)$ is established. Letting $\mathrm{R}\left(\mathrm{x}^{\prime}, \mathrm{y}^{\prime}, \mathrm{z}^{\prime}\right)=\left|\mathrm{r}^{\prime}\right|$, one has:

$$
\begin{aligned}
& q_{x}=x^{\prime} / R \\
& q_{y}=y^{\prime} / R \\
& q_{z}=z^{\prime} / R
\end{aligned}
$$

and $q_{x}{ }^{2}+q_{y}{ }^{2}+q_{z}{ }^{2}=1$. In the case at hand, $z^{\prime}=H_{o}$ is a constant and of interest is the relationship between $\mathrm{dx}^{\prime} \mathrm{dy} \mathrm{y}^{\prime}$ and $\mathrm{dq}_{\mathrm{x}} \mathrm{dq}_{\mathrm{y}}$. Using the Jacobian of the transformation to establish the required relationship, one obtains:

$$
\begin{aligned}
\mathrm{dq}_{\mathrm{x}} \mathrm{dq_{y }}= & \left|\begin{array}{cc}
\frac{\mathrm{dq_{x }}}{\mathrm{dx^{ \prime }}} & \frac{\mathrm{dq_{x }}}{d y^{\prime}} \\
\frac{d q_{y}}{d x^{\prime}} & \frac{d q_{y}}{d y^{\prime}}
\end{array}\right| \\
= & d x^{\prime} d y^{\prime}
\end{aligned}
$$

In establishing Equation D3 the following relationships have been used:

$$
\begin{aligned}
& \mathrm{dq}_{\mathrm{x}} / \mathrm{dx}=\left[1-(\mathrm{x} / \mathrm{R})^{2}\right] / \mathrm{R} \\
& \mathrm{dq}_{\mathrm{x}} / \mathrm{dy}=-(\mathrm{xy}) / \mathrm{R}^{3} \\
& \mathrm{dq}_{\mathrm{y}} / \mathrm{dx}=-(\mathrm{xy}) / \mathrm{R}^{3} \\
& \mathrm{dq}_{\mathrm{y}} / \mathrm{dy}=\left[1-(\mathrm{y} / \mathrm{R})^{2}\right] / \mathrm{R}
\end{aligned}
$$

The relationship between the direction cosines $\left(\mathrm{q}_{x}, \mathrm{q}_{\mathrm{y}}\right)$ and the spherical coordinates $(\theta, \phi)$ has also been used in the text. The coordinate transformation is:

$$
\begin{aligned}
& \mathrm{q}_{\mathrm{x}}=\cos \left(\alpha_{\mathrm{x}}\right)=\sin (\theta) \cos (\phi) \\
& \mathrm{q}_{\mathrm{y}}=\cos \left(\alpha_{\mathrm{y}}\right)=\sin (\theta) \sin (\phi)
\end{aligned}
$$


and the Jacobian of the transformation is:

$$
\text { Jacobian }=\left|\begin{array}{ll}
\frac{\mathrm{dq}_{\mathrm{x}}}{\mathrm{d} \theta} & \frac{\mathrm{dq}_{\mathrm{x}}}{\mathrm{d} \phi} \\
\frac{\mathrm{dq}_{\mathrm{y}}}{\mathrm{d} \theta} & \frac{\mathrm{dq}_{\mathrm{y}}}{\mathrm{d} \phi}
\end{array}\right|=\sin (\theta) \cos (\phi)
$$

Thus, one has

$$
\begin{aligned}
\mathrm{dq}_{\mathrm{x}} \mathrm{dq_{y }} & =\sin (\theta) \cos (\theta) \mathrm{d} \theta \mathrm{d} \phi \\
& =\cos (\theta) \mathrm{d} \Omega
\end{aligned}
$$

where $\mathrm{d} \Omega=\sin (\theta) \mathrm{d} \theta \mathrm{d} \phi$ is the differential "solid angle."

The inverse transformation is:

$$
d \Omega=\frac{d q_{x} d q_{y}}{\sqrt{1-q_{x}^{2}-q_{y}^{2}}}
$$




\section{APPENDIX E}

\section{RELATIONSHIP BETWEEN BRIGHTNESS AND ELECTRIC FIELD INTENSITY}

In the notation commonly used in radio astronomy (e.g., Kraus, 1966), the radiation from a source is defined in terms of the source "brightness." The brightness is defined to be the power per unit area arriving at the receiver per steradian from the direction $(\theta, \phi)$ and in a narrow frequency interval, $\Delta v$. In particular, $\mathrm{B}(\theta, \phi) \sin (\theta) \mathrm{d} \theta \mathrm{d} \phi=\mathrm{B}(\Omega) \mathrm{d} \Omega$ is the power per unit area per unit of bandwidth arriving at the receiver. The radiation received from a thermal source can be treated as if it were produced by electromagnetic fields on the surface of the source just as radiation from an antenna can be treated in terms of the equivalent electromagnetic fields on the aperture of the antenna. The objective of this appendix is to relate $B(\Omega)$ to these equivalent electromagnetic fields on the surface of the source.

In an electromagnetic system, power is obtained from the Poynting vector, $\underline{\mathbf{P}}=\underline{\mathrm{E}} \times \underline{\mathrm{H}}$. When the observer is very far from the source, the electromagnetic fields are nearly plane waves and the magnitude of the Poynting vector is approximately: $\mathrm{P}=\underline{\mathrm{E}} \cdot \underline{\mathrm{E}} / \mathrm{Z}_{\mathrm{o}}$ where $Z_{\mathrm{o}}=\sqrt{\mu_{\mathrm{o}} / \epsilon_{\mathrm{o}}}$ is the characteristic impedance of free space. Assuming that the fields are ergodic, stationary random processes (in the time coordinate), one obtains:

$$
\begin{aligned}
\langle\mathrm{P}(\mathrm{r}, \mathrm{t})\rangle & =\langle\underline{\mathrm{E}}(\underline{\mathrm{r}}, \mathrm{t}) \cdot \underline{\mathrm{E}}(\underline{\mathrm{r}}, \mathrm{t})\rangle / \mathrm{Z}_{\mathrm{o}} \\
& =\int_{-\infty}^{\infty} \mathrm{P}(\underline{\mathrm{r}}, \mathrm{v}) \mathrm{dv}
\end{aligned}
$$

where $P(\underline{r}, v)$ is the Fourier transform of the autocorrelation function $\langle\underline{E}(\underline{r}, t) \cdot \underline{E}(\underline{r}, t+\tau)\rangle$ evaluated at the position $\underline{r}$. It can also be expressed in terms of the Fourier-Stieltjes transform, $\underline{E}(\underline{r}, v)$, in the form: $P(\underline{r}, v)=\left\langle\underline{E}(\underline{r}, v) \cdot \underline{E}^{*}(r, v)>/ Z_{0}\right.$. It follows from Equation $E 1$ that the time average power in a frequency band, $\Delta v$, which is narrow compared to $P(r, v)$ is approximately $\mathrm{P}(\underline{\mathrm{r}}, \mathrm{v}) \Delta \mathrm{v}$ (e.g., Papoulis, 1965).

The spectrum $P(\underline{r}, v)$ of the radiation at the receiver at $\underline{r}$ due to a patch of surface at $\underline{r}^{\prime}$ can be calculated using the procedure outlined in Appendix $B$ (letting $i=j$ ). Assuming that the radiation separates in space and time coordinates and is spatially uncorrelated, one obtains:

$$
P(\underline{r}, v) d \underline{r}^{\prime}=\left[H_{0} / R^{2}\left(\underline{r} / \underline{r}^{\prime}\right)\right]^{2}\left[1 / \lambda^{2}\right]<\underline{E}_{1}\left(\underline{r}^{\prime}, v\right) \cdot \underline{E}_{1}\left(\underline{r}^{\prime}, v\right) / Z_{0}>_{p} d \underline{r}^{\prime}
$$


Comparing this expression with the definition of brightness, one obtains:

$$
B(\Omega) d \Omega=P(\underline{r}, v) d \underline{r}^{\prime}=\left[1 / \lambda^{2}\right]<\underline{E}_{t}\left(\underline{r}^{\prime}, v\right) \cdot \underline{E}_{1}\left(\underline{r}^{\prime}, v\right)>/ Z_{0}>_{p}\left[H_{o} / R^{2}\left(\underline{r} / \underline{r}^{\prime}\right)\right]^{2} d \underline{r}^{\prime}
$$

Changing from Cartesian to spherical coordinates on the right-hand side in Equation E3, one has $\left[\mathrm{H}_{\mathrm{o}} / \mathbf{R}^{2}\left(\mathbf{r} / \underline{\mathbf{r}}^{\prime}\right)\right]^{2} \mathrm{~d} \underline{\underline{r}}^{\prime}=$ $\cos (\theta) \mathrm{d} \Omega$. Hence, the relationship between the brightness and the electromagnetic fields on the surface is:

$$
\mathrm{B}(\Omega)=\left[1 / \lambda^{2}\right]\left[<\underline{\mathrm{E}}_{\mathrm{t}}(\Omega, \mathrm{v}) \cdot \underline{\mathrm{E}}_{\mathrm{t}}(\Omega, \mathrm{v})>_{\mathrm{p}} / \mathrm{Z}_{\mathrm{o}}\right] \cos (\theta)
$$

The brightness can also be expressed in terms of the direction cosines $\left(q_{x}, q_{y}\right)$. Defining an alternative brightness, $\widetilde{B}(q)$, and noting that (Appendix D) $\mathrm{dq}_{\mathrm{x}} \mathrm{dq}_{\mathrm{y}}=\left(\mathrm{H}_{\mathrm{o}} / \mathrm{R}_{\mathrm{o}}{ }^{2}\right)^{2} \mathrm{dx} \mathrm{dy}^{\prime}$, one obtains:

$$
\widetilde{\mathrm{B}}(\underline{\mathrm{q}}) \mathrm{dq}=\mathrm{P}(\underline{\mathrm{r}}, \mathrm{v}) \underline{\mathrm{dr}}^{\prime}=\left[1 / \lambda^{2}\right]\left[\left\langle\underline{\mathrm{E}}_{\mathrm{t}}(\underline{\mathrm{q}}, \mathrm{v}) \cdot \underline{\mathrm{E}}_{\mathrm{t}}(\underline{\mathrm{q}}, \mathrm{v})\right\rangle_{\mathrm{p}} / \mathrm{Z}_{\mathrm{o}}\right] \mathrm{dq}
$$

Finally, note that

$$
\widetilde{\mathrm{B}}(\underline{\mathrm{q}})=\mathrm{B}(\Omega) / \cos (\theta)=\mathrm{B}(\underline{\mathrm{q}}) / \sqrt{1-\mathrm{q}^{2}-\mathrm{q}_{\mathrm{y}}{ }^{2}}
$$

\section{REFERENCES:}

Kraus, J.D. (1966), Radio Astronomy, McGraw-Hill Inc.

Papoulis, A. (1965), Probability, Random Variables, and Stochastic Processes, Chapter 10, McGraw-Hill, Inc. 


\section{APPENDIX F}

\section{THE SYNTHESIZED BEAM}

The synthesized beam $G\left(\underline{q}_{m n}\right)$ as defined in the text (Equation 24a) is:

$$
\mathrm{G}\left(\underline{\mathrm{q}}_{\mathrm{m}, \mathrm{n}}-\underline{\mathrm{q}}\right)=\operatorname{DFT}[\mathrm{W} \exp (-j 2 \pi \underline{\eta} \underline{\mathrm{q}})]
$$

where DFT denotes a discrete Fourier transform in two dimensions on the variables $\left(\eta_{x}, \eta_{y}\right)$ and $w$ is an arbitrary

"weight" added here to account for biases in the measurements (e.g., differences in the effective time-bandwidth product or redundancies) or to permit beam shaping to control side lobes. Since the exponential is separable in $\underline{\eta}$, the synthesized beam $G\left(\underline{q}_{m n}-\underline{q}\right)$ also separates and can be factored into the product of two functions: $G\left(\underline{q}_{m n}-\underline{q}\right)=G_{m}\left(q_{m}-q_{x}\right)$. $G_{n}\left(q_{n}-q_{y}\right)$. Each of the $G_{m, n}\left(q_{m, n}-q_{x, y}\right)$ is a one-dimensional, discrete Fourier transform of the exponential $\exp \left(-j 2 \pi \eta_{m, n} q_{x, y}\right)$. Using the following definition of the discrete Fourier transform

$$
\text { DFT[ F ] }=(1 / M) \sum_{s=\{M\}} F(s) \exp (j 2 \pi[k / M] s)
$$

each of the one-dimensional transforms can be written as follows:

$$
\begin{aligned}
& G_{m}\left(q_{m i}-q_{x}\right)=(1 / M) \sum_{s=\{M\}} W_{s} \exp \left(j 2 \pi\left[(m / M) s-\eta_{x} q_{x}\right]\right) \\
& G_{n}\left(q_{n}-q_{y}\right)=(1 / N) \sum_{s=\{N\}} Y_{s} \exp \left(j 2 \pi\left[(n / N) s-\eta_{y} q_{y}\right]\right)
\end{aligned}
$$

Equations F3 can be evaluated explicitly in the important, special case when the measurements are made at (or can be mapped onto) baselines which are multiples of some minimum spacing $d_{x}$ and $d_{y}$ in each dimension. The sums in Equa- 
tions $\mathrm{F} 3$ can be evaluated in closed form in this case and the results will be given below. However, since the sums are identical, except for an exchange of subscripts, the discussion below will be for only one term, $G_{m}\left(q_{m}-q_{x}\right)$.

When the measurements are made at multiples of some minimum spacing, $d_{x}$, the parameter $\eta_{x}(s)$ becomes an integer multiple of $\left(d_{x} / c\right) v_{o}=d_{x} / \lambda$ (e.g., see Equation B11, Appendix B). In this case, Equations F3 have the form:

$$
\mathrm{G}_{\mathrm{m}}\left(\mathrm{q}_{\mathrm{m}}-\mathrm{q}_{\mathrm{x}}\right)=(1 / \mathrm{M}) \sum_{\mathrm{s}=\{\mathrm{M}\}} \mathrm{w}_{\mathrm{s}} \exp \left(\mathrm{jk}_{\mathrm{o}} \mathrm{d}_{\mathrm{x}}\left[\mathrm{m} \lambda /\left(\mathrm{Md}_{\mathrm{x}}\right)-\mathrm{q}_{\mathrm{x}}\right] \mathrm{s}\right)
$$

where $k_{0}=2 \pi / \lambda$. Comparing the right- and left-hand sides, it is clear that $q_{m}=m \lambda /\left(M_{x}\right)$ where $M$ is the number of terms in the sum.

In order to proceed further, consider the special case when all of the weights, $\mathrm{W}_{\mathrm{s}}$, are equal. In this case, the sum in Equation F4 can be evaluated explicitly (it is a geometrical progression) and one obtains:

$$
\begin{aligned}
G_{m}\left(q_{m}-q_{x}\right)= & \frac{1}{M} \frac{\sin \left[\pi M d_{x}\left(q_{m}-q_{x}\right) / \lambda\right]}{\sin \left[\pi d_{x}\left(q_{m}-q_{x}\right) / \lambda\right]} \\
& =\frac{\operatorname{sinc}\left[M d_{x}\left(q_{m}-q_{x}\right) / \lambda\right]}{\operatorname{sinc}\left[d_{x}\left(q_{m}-q_{x}\right) / \lambda\right]}
\end{aligned}
$$

where $\operatorname{sinc}(x)=\sin (\pi x) / x$. Notice that Equation F5 is just the array factor of a linear array of M identical antenna elements with uniform spacing, $d_{x}$ (Jordan and Balmain, 1968).

Notice that there are $M$ terms in the sum in Equations $F 3$ and $F 4$ and, therefore, also $M$ possible values of $q_{m}$. The $\mathrm{M}$ terms consist of measurements at $\hat{M}$ baselines, the complex conjugate of these measurements and a measurement at the zero baseline. Hence, one has $M=2 \hat{M}+1$ terms in the summation in Equation $F 4$. The values of the index $m$ are: $m$ $=0, \pm 1, \pm 2 \ldots \pm \hat{M}$. Also, notice that the physical length of the synthesis array (i.e., the maximum baseline) is $\hat{\mathrm{M}} \mathrm{d}_{\mathrm{x}}$; but the equivalent electrical length as compared to a linear antenna array with the same resolution is $\operatorname{Md}_{\mathrm{x}}$.

Also, notice that each value of $m$ will result in a data point (pixel) in the image ( $M$ points in the $x$-dimension and $N$ points in the $y$-dimension). The increment between these data points in direction cosine space is $q_{m}$. However, these points are not separated by a full synthesized beam width. To see this, define the resolution of the synthesized beam to be 
the distance between the first nulls on each side of the main lobe. Denoting this distance by $q_{0}$, it is clear from Equation F5 that:

$$
\mathrm{q}_{\mathrm{o}}=2 \lambda / \mathrm{Md}_{\mathrm{x}}=\lambda / \mathrm{D}_{\mathrm{x}}
$$

where $D_{x}=M d_{x}$ is the physical length of the array used to synthesize the beam (i.e., the maximum baseline). Since -1 $<\mathrm{q}_{\mathrm{x}}<+1$ it follows that there are $2 / \mathrm{q}_{\mathrm{o}}=\mathrm{Md}_{\mathrm{x}} / \lambda=2 \mathrm{D}_{\mathrm{x}} / \lambda$ contiguous resolution cells in the image. On the other hand, there are a total of $M$ pixels in the reconstructed image in each dimension and each pixel is separated by $q_{m}=\lambda /\left(M_{x}\right)$ (Equation F4). Since $q_{m}=q_{0} / 2$ it follows that there are two pixels per resolution cell.

Finally, notice that with uniform weight, grating lobes will appear in the image at integer multiples of $\lambda / d_{x}=q_{x}$ (Equation F5). Since $-1<q_{x}<+1$, one must have $\lambda>d_{x}$ in order to avoid the presence of grating lobes in the field of view of the sensor.

\section{REFERENCES:}

Jordan, E.C. and K.G. Balmain (1968), Electromagnetic Waves and Radiating Systems, Chapter 12, Prentice-Hall, Englewood Cliffs, New Jersey. 


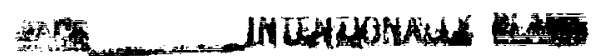




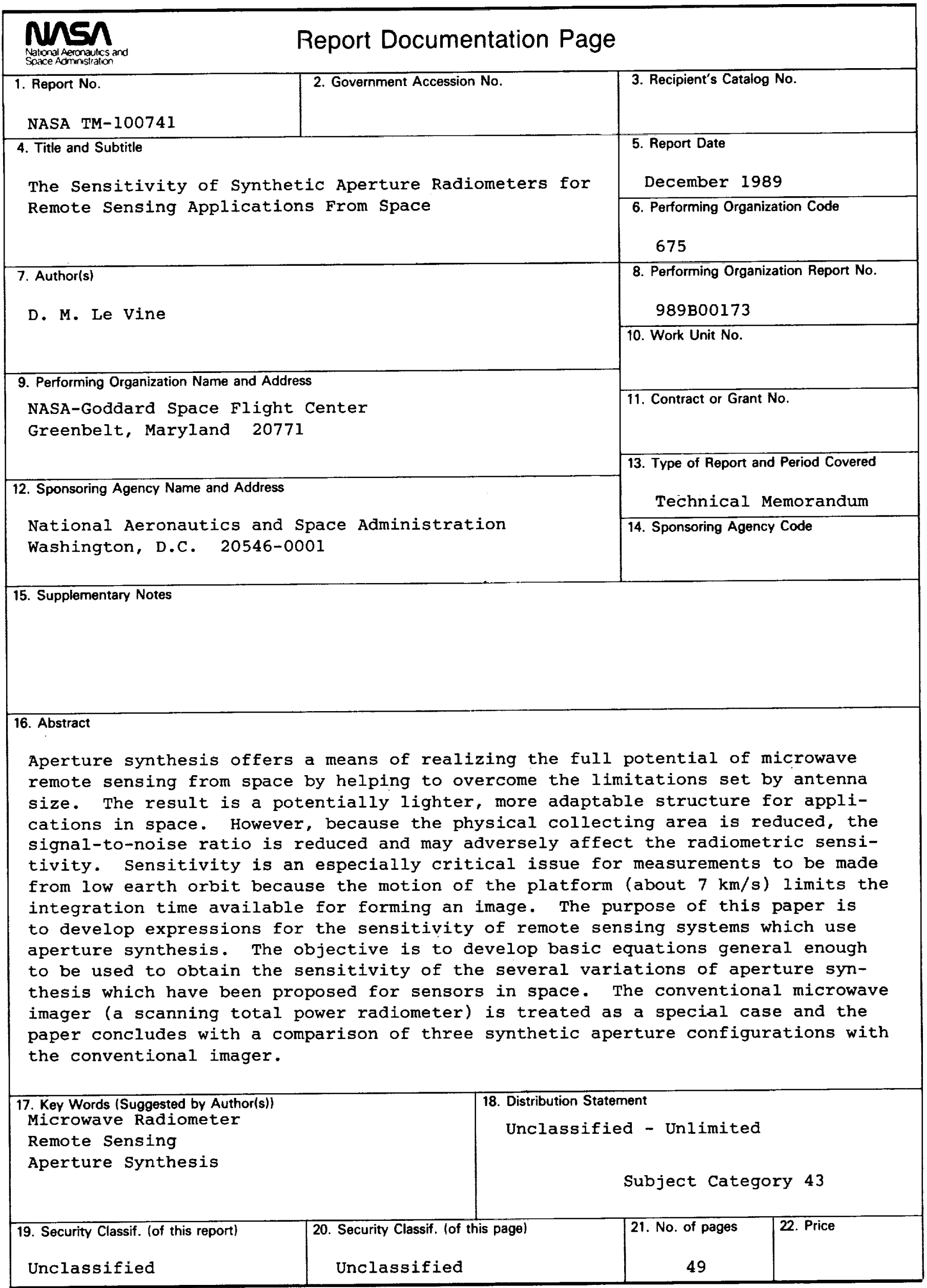


-5) 